Florida International University FIU Digital Commons

7-21-2003

\title{
Cross-section measurements for the near ultra- violet absorption spectra of glyoxal at the 280 nanometer wavelength
}

Abigail Diaz

Florida International University

DOI: $10.25148 /$ etd.FI14062268

Follow this and additional works at: https://digitalcommons.fiu.edu/etd

Part of the Chemistry Commons

\section{Recommended Citation}

Diaz, Abigail, "Cross-section measurements for the near ultra-violet absorption spectra of glyoxal at the 280 nanometer wavelength" (2003). FIU Electronic Theses and Dissertations. 2798.

https://digitalcommons.fiu.edu/etd/2798 
FLORIDA INTERNATIONAL UNIVERSITY

Miami, Florida

CROSS-SECTION MEASUREMENTS FOR THE NEAR ULTRA-VIOLET ABSORPTION SPECTRA OF GLYOXAL AT THE 280 NANOMETER WAVELENGTH

A thesis submitted in partial fulfillment of the requirements for the degree of MASTER OF SCIENCE in

CHEMISTRY

by

Abigail Diaz 
To: Dean Arthur W. Herriott

College of Arts and Sciences

This thesis, written by Abigail Diaz, and entitled CrossSection Measurements for the Near Ultra-Violet Absorption Spectra of Glyoxal at the 280 Nanometer wavelength, having been approved in respect to style and intellectual content, is referred to you for judgment.

We have read this thesis and recommend that it be approved.

David Chatfield

James M. Quirke

Jeffrey A. Joens, Major Professor

Date of Defense: July 21, 2003

The thesis of Abigail Diaz is approved.

Dean Arthur w. Herriott College of Arts and Sciences

Dean Douglas Wartzok University Graduate School

Florida International University, 2003 


\section{ACKNOWLEDGMENTS}

I would like to thank Dr. Jeffrey Joens for his guidance and assistance during my research. I wish to also thank Dr. David Chatfield for his advice and aid in the development of my improved understanding of computational chemistry. A special token of gratitude goes to Dr. Palmer Graves, who supported me every step of the way.

Finally, I want to express my thanks to the chemistry faculty for their support and the part they played in my intellectual enrichment. 


\section{ABSTRACT OF THE THESIS}

CROSS-SECTION MEASUREMENTS FOR THE NEAR ULTRA-VIOLET ABSORPTION SPECTRA OF GLYOXAL AT THE 280 NANOMETER WAVELENGTH

by

\section{Abigail Diaz}

Florida International University, 2003 Miami, Florida

Professor Jeffrey A. Joens, Major Professor

Glyoxal and other dicarbonyl compounds have been detected in tropospheric air in rural areas and polluted urban areas. Accurate absorption cross-sections for the near UV spectrum of glyoxal are important since the value of this chemical characteristic is necessary for calculating effective quantum yields and ultimately the rates and products of photolysis. In this study, comprehensive measurements were made for the $280 \mathrm{~nm}$ gasphase absorption spectrum of glyoxal. In addition, molecular orbital calculations were carried out and the results used as a guide to identify the vibration responsible for the weak structure features of the continuous area of the glyoxal spectrum. That vibration was found to be an $a_{g}$ co stretch vibration mode of the glyoxal molecule. 
I. INTRODUCTION

Properties of glyoxal

Atmospheric chemistry of glyoxal .

Molecular orbital energy level diagram

Review of previous work

II. EXPERIMENTAL DATA ANALYSIS

Preparation of glyoxal

Apparatus .

Test of the apparatus

Wavelength calibration

Spectral resolution.

Data acquisition and analysis

Data analysis

Cross-section calculation

III. EXPERIMENTAL RESULTS

Summary of results

Comparison with previous results

IV. COMPUTATIONAL THEORETICAL BACKGROUND

The Schodinger equation

The Born-Oppenheimer approximation.

Hartree-Fock theory .

Electron spin

Basis sets

Basis set effects

Diffuse functions

Electron correlation.

Configuration interation

Gaussian

Gaussian input .

V. GROUP THEORY

Molecular vibrations

Fundamental vibrational frequencies for

Allowed transitions.

VI. ANALYSIS OF GAUSSIAN CALCULATIONS

Job output analysis.

First excited state. 
Molden . $\quad . \quad . \quad . \quad . \quad . \quad . \quad .65$

Evaluation of CIS performance. $\quad . \quad$. $\quad .67$

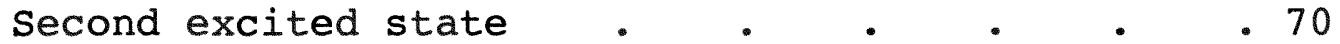

Third excited state. $\quad . \quad . \quad . \quad . \quad 073$

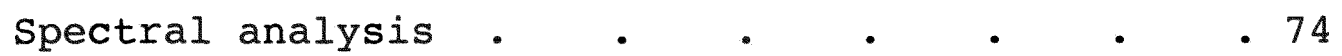

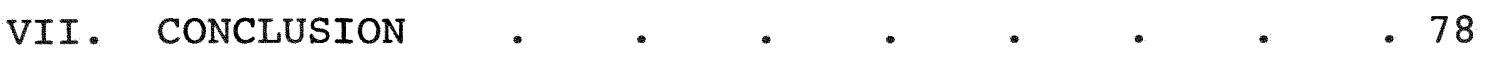

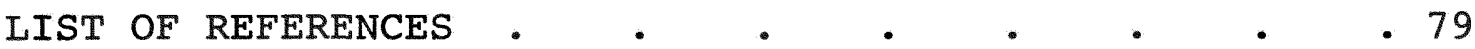

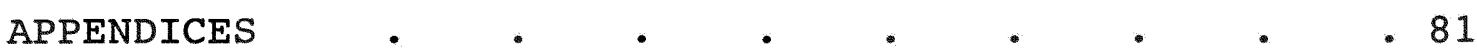




\section{LIST OF TABLES}

TABLE

PAGE

TABLE 1.1 - Yield of Glyoxal from the Oxidation of Benzene, Toluene and p-xylene • . . 6

TABLE 2.1 - Comparison of Benzene Location Peaks • . 27

TABLE 2.2 - Location of Benzene Peaks at $1 \mathrm{~nm}$ Bandwidth. 28

TABLE 2.3 - Summary of the Data Analysis Process • . 31

TABLE 2.4 - Operating Conditions for the Spectrophotometer $\quad \cdot \quad \cdot \quad \cdot \quad \cdot \quad$. 29

TABLE 3.1 - Comparison of Previous Cross-Section Values $\left(\mathrm{cm}^{2} /\right.$ molecule) $\quad . \quad . \quad . \quad . \quad \cdot \quad \cdot 37$

TABLE 4.1 - Outline of Gaussian Input . . . . 54

TABLE 5.1 - Fundamental Frequencies for Trans-Glyoxal • 60

TABLE 6.1 - Results of the Frequency $\left(\mathrm{cm}^{-1}\right)$ Calculations For the First Excited State of

Trans-Glyoxal Using the CIS Method . . 66

TABLE 6.2 - Results of the Frequency $\left(\mathrm{cm}^{-1}\right)$ Calculations For the First Excited state of

Trans-Glyoxal Using the $6-311++G(D, P)$

CIS Method $• \quad \cdot \quad \cdot \quad \cdot \quad \cdot \quad \cdot \quad \cdot 68$

TABLE 6.3 - Results by Stanton and Gauss [1997] for the Frequency $\left(\mathrm{cm}^{-1}\right)$ Calculations for the Ground and First Excited State of Trans-Glyoxal Using the EOMEE-CCSD

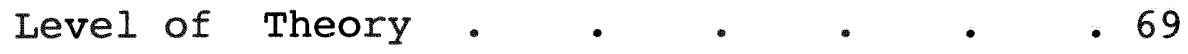

TABLE 6.4 - Results of the Frequency $\left(\mathrm{cm}^{-1}\right)$ Calculations For the Second Excited State of Trans-Glyoxal Using the CIS Method • • 71

TABLE 6.5 - Results of the Frequency $\left(\mathrm{cm}^{-1}\right)$ Calculations for the Third Excited state of Trans-Glyoxal Using the CIS Method • . 73 
TABLE 6.6 - Results by Osamura et al. [1981] of the Frequency Calculations for the $\mathrm{C}_{\mathrm{s}}$

Stationary Point Connecting Glyoxal

with $\mathrm{H} 2 \mathrm{CO}+\mathrm{CO} \quad . \quad . \quad . \quad . \quad 75$

TABLE 6.7 - Spacings for the Continuous Region of the Glyoxal Spectra at the $280 \mathrm{~nm}$ Wavelength .76 


\section{LIST OF FIGURES}

FIGURE

PAGE

FIGURE 1.1 - Drawing of trans-glyoxal molecule. . . 3

FIGURE 1.2 - Initial steps of the $\mathrm{OH}$ radical initiated oxidation of toluene . . . . . 5

FIGURE 1.3 - Possible pathways for the formation of glyoxal from toluene • • • • • 7

FIGURE 1.4 - Molecular orbitals for glyoxal • . . 10

FIGURE 1.5 - Gas-phase absorption spectra determined for glyoxal by Plum et al. . . . . 12

FIGURE 2.1 - Schematic diagram of the gas handling manfold $\quad . \quad . \quad . \quad . \quad . \quad 17$

FIGURE 2.2 - Distillation apparatus . . . . 21

FIGURE 2.3 - Infrared spectrum of glyoxal . . . 22

FIGURE 2.4 - Infrared spectrum of benzene . . . 23

FIGURE 2.5 - Schematic of the experimental apparatus - 24

FIGURE 2.6 - Absorbance vs. concentration for the potassium dichromate calibration solution . 25

FIGURE 2.7 - Absorbance vs. pressure for glyoxal at 1) $330 \mathrm{~nm}, 2) 310 \mathrm{~nm}$ and 3) $270 \mathrm{~nm}$. 33

FIGURE 3.1 - Spectra of glyoxal . . . . . 36 


\section{Chapter I}

\section{Introduction}

Dicarbonyl compounds such as glyoxal ( $\mathrm{CHOCHO}$ ) and methylglyoxal $\left(\mathrm{CH}_{3} \mathrm{C}(\mathrm{O}) \mathrm{CHO}\right)$ have been measured in tropospheric air, in rural areas at small concentrations, and in polluted urban areas. Munger et al. [1995] report an average concentration of 44 ppt glyoxal in Central Virginia, and Lee et al. [1995] measured average glyoxal concentrations of 18-83 ppt and average methylglyoxal concentrations of 31-88 ppt at a rural site in Georgia. [Finlayson-Pitts and Pitts, 2000] Higher concentrations are found in urban air, for example, $0.78 \pm 0.85 \mathrm{ppb}$ glyoxal and $1.0 \pm 0.6 \mathrm{ppb}$ methylgloxal in the Los Angeles area. [Finlayson-Pitts and Pitts, 2000]

There is a growing interest as well as need to investigate the atmospheric loss processes of glyoxal and other dicarbonyl compounds in more detail. Accurate absorption cross-sections for the near UV spectra of these two molecules are important since the value of this chemical characteristic is necessary for calculating effective quantum yields and ultimately the rates and products of photolysis. Moreover, cross-section data are also of use for laboratory studies of glyoxal chemistry 


\section{Chapter I}

\section{Introduction}

Dicarbonyl compounds such as glyoxal (CHOCHO) and methylglyoxal $\left(\mathrm{CH}_{3} \mathrm{C}(\mathrm{O}) \mathrm{CHO}\right)$ have been measured in tropospheric air, in rural areas at small concentrations, and in polluted urban areas. Munger et al. [1995] report an average concentration of 44 ppt glyoxal in Central Virginia, and Lee et al. [1995] measured average glyoxal concentrations of 18-83 ppt and average methylglyoxal concentrations of 31-88 ppt at a rural site in Georgia. [Finlayson-Pitts and Pitts, 2000] Higher concentrations are found in urban air, for example, $0.78 \pm 0.85 \mathrm{ppb}$ glyoxal and $1.0 \pm 0.6 \mathrm{ppb}$ methylgloxal in the Los Angeles area. [Finlayson-Pitts and Pitts, 2000]

There is a growing interest as well as need to investigate the atmospheric loss processes of glyoxal and other dicarbonyl compounds in more detail. Accurate absorption cross-sections for the near UV spectra of these two molecules are important since the value of this chemical characteristic is necessary for calculating effective quantum yields and ultimately the rates and products of photolysis. Moreover, cross-section data are also of use for laboratory studies of glyoxal chemistry 
where glyoxal concentration is determined by absorption spectroscopy

There are presently several inconsistencies in the published measurements of absorption cross-sections of glyoxal, as is discussed below.

In the present work, comprehensive measurements have been made for the gas phase absorption spectrum of glyoxal. The results from these measurements can be used to estimate the lifetime of glyoxal with respect to photodissociation for a representative set of tropospheric conditions.

In addition, molecular orbital calculations were also carried out and the results compared to what was observed experimentally and to previous results. The molecular orbital calculations were also used as a guide to identify the vibration responsible for the weak structured features of the continuous area of the spectrum.

\section{Properties of glyoxal}

Glyoxal is an organic molecule with a molecular formula of $\mathrm{C}_{2} \mathrm{H}_{2} \mathrm{O}_{2}$. (See Figure 1.1) Glyoxal is a powerfully reducing and reactive solid (yellow prisms or irregular pieces turning white on cooling) with a melting point of $15^{\circ} \mathrm{C}$ and of high vapor pressure and low boiling point of $50^{\circ} \mathrm{C}$. [Bretherick, 1985] Mixtures of glyoxal with air may explode, and contact with water causes violent 


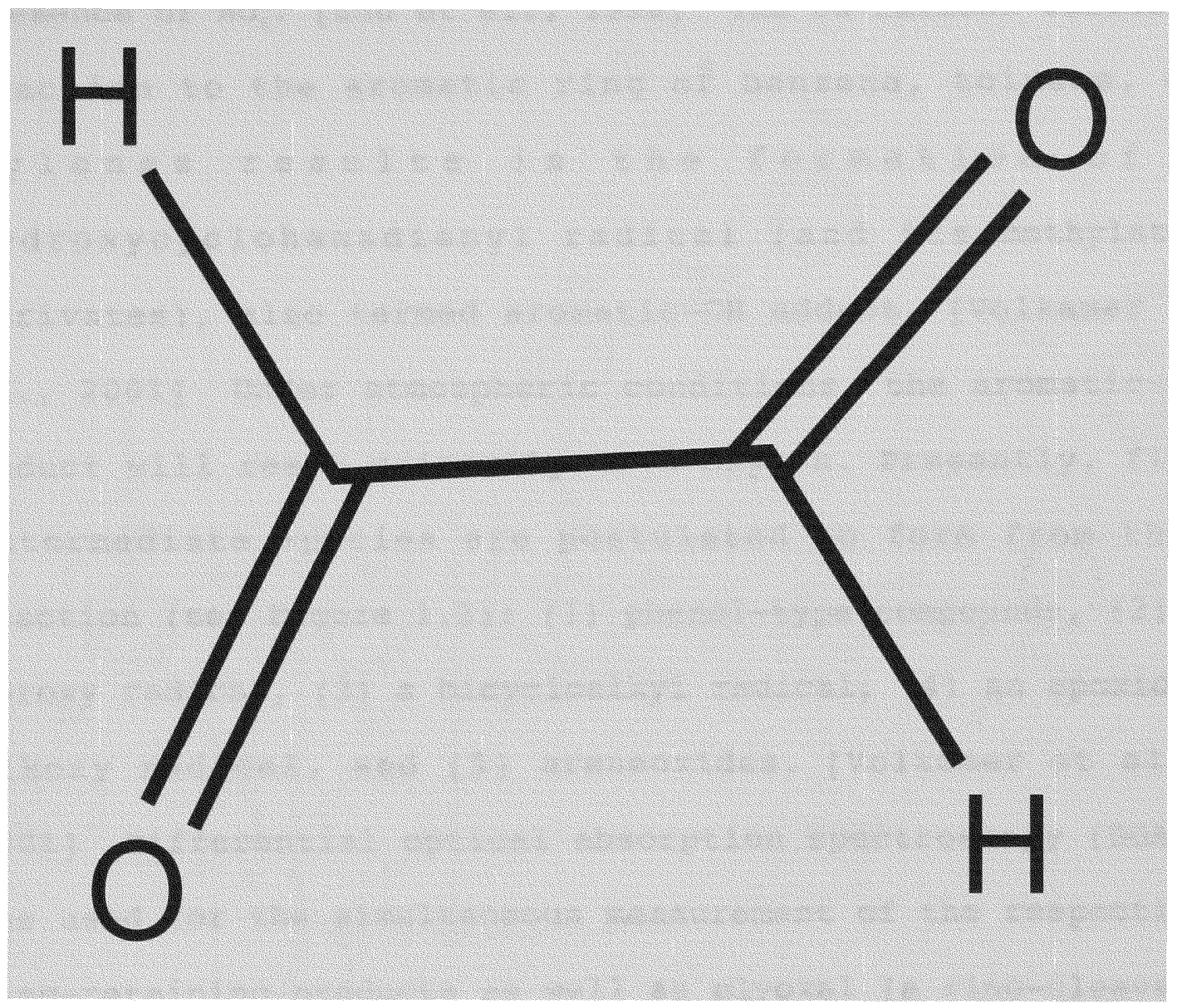

Figure 1.1. Drawing of trans-glyoxal molecule.

polymerization. [Bretherick, 1985] Like formaldehyde, pure glyoxal may polymerize exothermally and ignite in storage. When heated to decomposition it emits acrid smoke and irritating vapors. [Lewis, 1997]

Atmospheric chemistry of glyoxal

Glyoxal is an important ring-cleavage product in the air photo-oxidation of some aromatic hydrocarbons in the 
presence of $\mathrm{NO}_{\mathrm{x}}$. [ $\mathrm{zhu}$ et al., 1996] The $\mathrm{OH}$ radical addition reaction to the aromatic ring of benzene, toluene, or xylenes results in the formation of a hydroxycyclohexadienyl radical (and its methylated derivates], also termed aromatic-OH adduct. [Volkamer et al., 2001] Under atmospheric conditions, the aromatic-OH adduct will react primarily with oxygen. Presently, five intermediate species are postulated to form from this reaction (see Figure 1.2): (1) phenol-type compounds, (2) a peroxy radical, (3) a bicycloalkyl radical, (4) an epoxidealkoxy radical, and (5) areneoxides. [Volkamer et al., 2001] Differential optical absorption spectroscopy (DOAS) was used for the simultaneous measurement of the respective ring-retaining products as well as glyoxal (a ring-cleavage product) in a series of experiments at the EUPHORE, large outdoor simulation chamber in Valencia, Spain. [Volkamer et al., 2001] The ring-retaining products and glyoxal were identified as primary products. According to this study, the yield of glyoxal was determined to be $35 \% \pm 10 \%$ from benzene and about 5\% higher from toluene and p-xylene. [Volkamer et al., 2001] They noted that the error of the absolute glyoxal yield determined in their study is dominated by the uncertainty of the UV absorption crosssections of glyoxal and that an improved determination is 


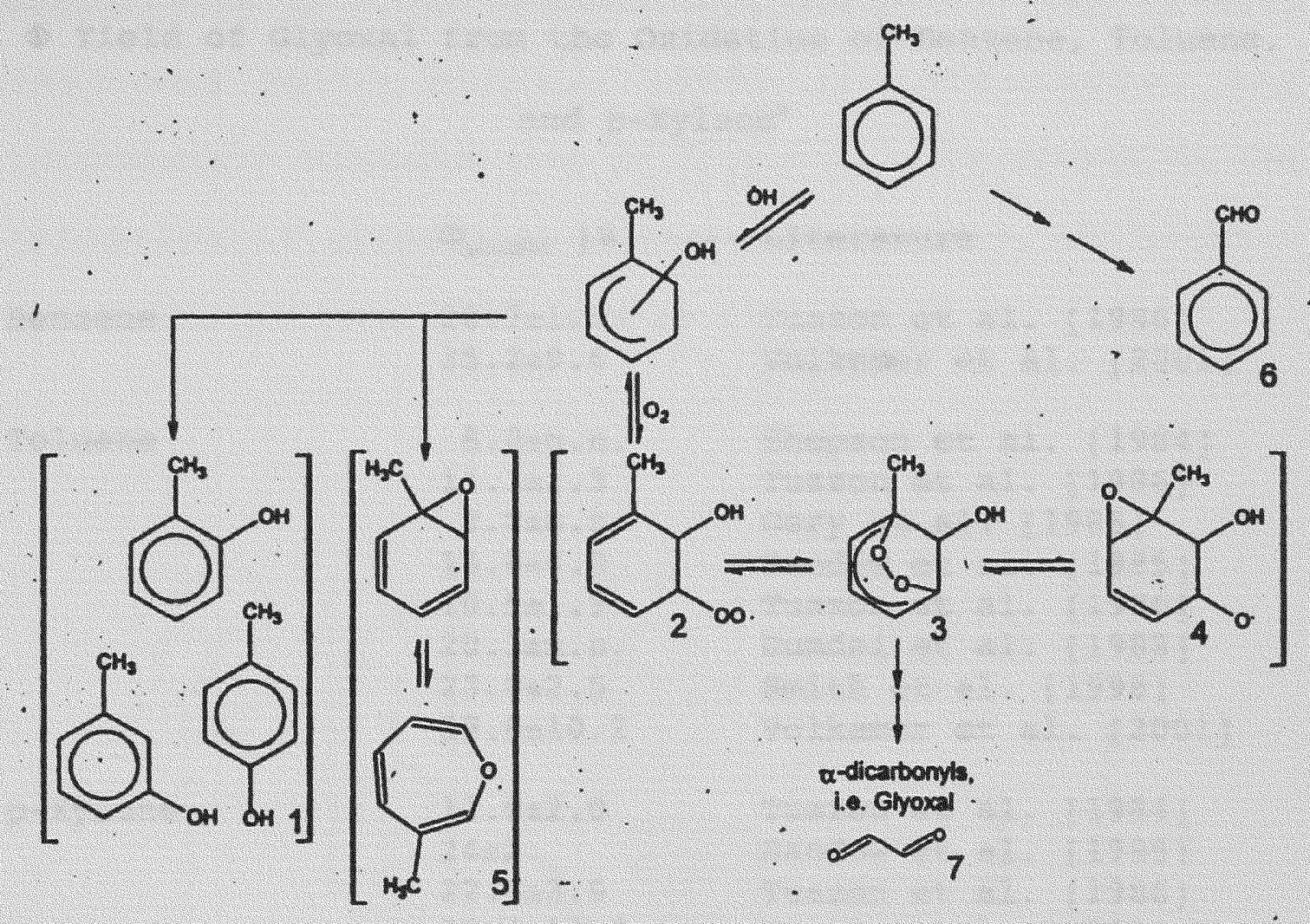

Figure 1.2. Initial steps of the $\mathrm{OH}$ radical initiated oxidation of toluene. Analogous intermediates are proposed for benzene and p-xylene. [Volkamer et al., 2001]

desirable. A comparison of glyoxal yields for of and the aforementioned aromatic compounds of previous years can be seen in Table 1.1. [Volkamer et al., 2001]

The additional yet negligible contribution of pathways forming glyoxal through the $\mathrm{OH}$ reaction of stable intermediate compounds is due to the dominant photolysis of 
$\Phi$ Yield of Glyoxal from the Oxidation of Benzene, Toluene, and $\mathrm{p}-\mathrm{xy}$ lene

\begin{tabular}{|c|c|c|}
\hline & $\Phi_{\text {product }}[\%]$ & Literature \\
\hline Benzene & $\begin{array}{l}20.7 \pm 1.9 \\
35.2 \pm 9.6\end{array}$ & $\begin{array}{l}\text { Tuazon et al. [1986] } \\
\text { Volkamer et al. [2001] }\end{array}$ \\
\hline Toluene & $\begin{array}{c}8 \cdot 0 \pm n \cdot n \\
11 \cdot 1 \pm 1 \cdot 3 \\
9 \cdot 8 \pm n \cdot n \\
15 \cdot 0 \pm 4 \cdot 0 \\
10 \cdot 5 \pm 1 \cdot 9 \\
20 \cdot 3 \pm n \cdot n . \\
23 \cdot 8 \pm 2 \cdot 5 \\
39 \cdot 0 \pm 10 \cdot 2\end{array}$ & $\begin{array}{l}\text { Shepson et al. [1984] } \\
\text { Tuazon et al. [1986] } \\
\text { Gery et al. [1985] } \\
\text { Bandow et al. [1985] } \\
\text { Tuazon et al. [1986] } \\
\text { Dumdei et al. [1988] } \\
\text { Smith et al. [1998] } \\
\text { Volkamer et al. [2001] }\end{array}$ \\
\hline p-xylene & $\begin{array}{l}12.0 \pm 2.0 \\
24 \pm 2 \\
22.5 \pm 3.9 \\
39.4 \pm 11.0 \\
40.4 \pm 10.6\end{array}$ & $\begin{array}{l}\text { Tuazon et al. [1984] } \\
\text { Bandow et al. [1985] } \\
\text { Tuazon et al. [1986] } \\
\text { Smith et al. [1999] } \\
\text { Volkamer et al. [2001] }\end{array}$ \\
\hline
\end{tabular}

a) Volkamer et al. [2001]

potential precursors for secondary glyoxal, i.e. unsaturated 1,4-dicarbonyl-type products. Formation of butenedial from toluene and o-xylene, 4-oxo-2-pentenol from toluene and o- and m-xylene, and 3-hexene-2,5-dione from pxylene and $1,2,4$-trimethylbenzene have all been experimentally observed and are postulated as ring cleavage 
carbonyl products during the oH-initiated atmospheric degradation of those aromatic hydrocarbons. [Lui et al., 1999 and Smith et al., 1999] In principle, any intermediate compound seen in Figure 1.3 may result in the formation of glyoxal as primary, secondary, or highergeneration product. [Volkamer et al., 2001]

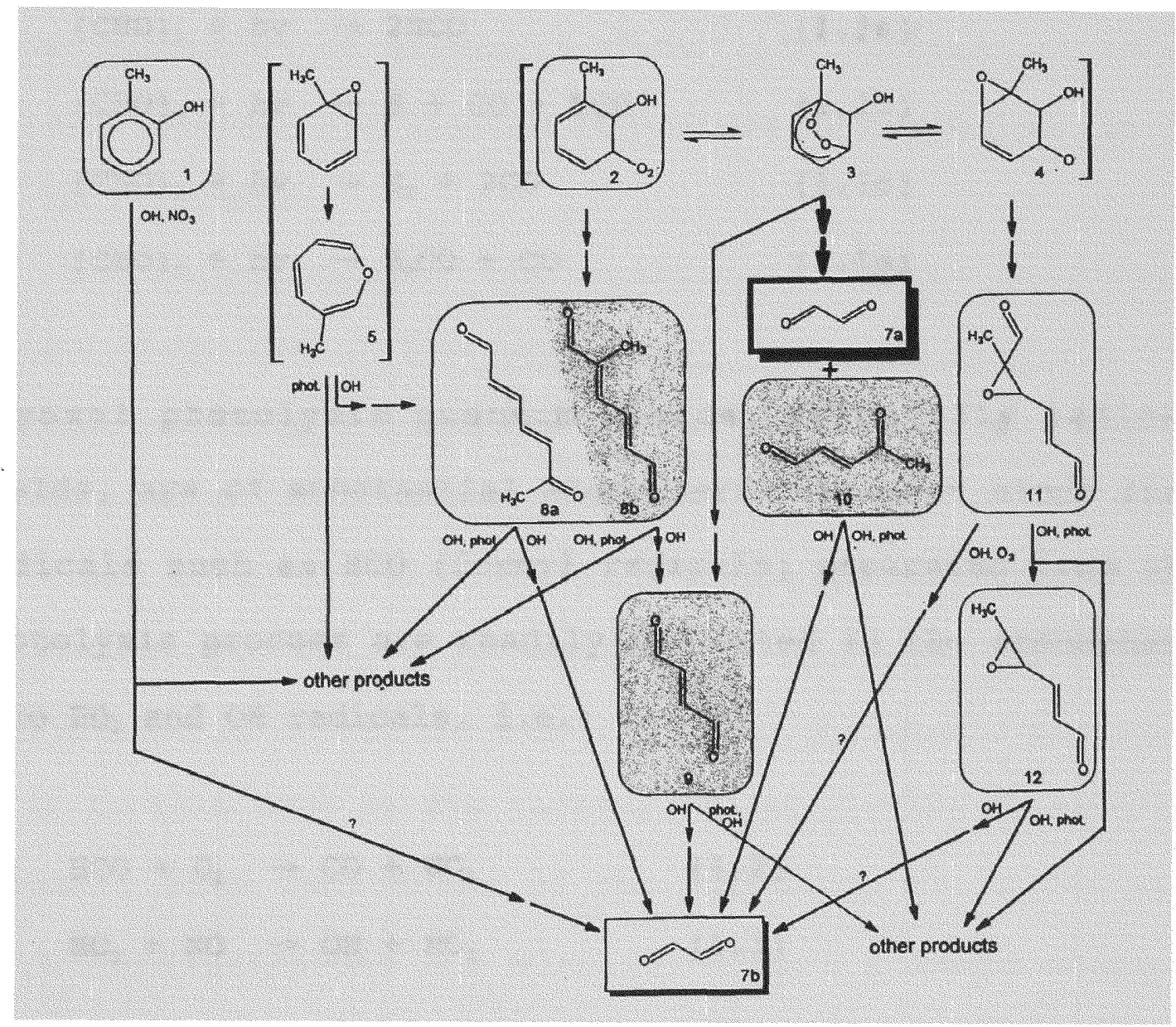

Figure 1.3. Possible pathways for the formation of glyoxal (shaded boxes) from toluene. [Volkamer et al., 2001] 
Some of the loss processes of glyoxal are known to be reaction with $\mathrm{OH}$ radicals and scavenging by atmospheric droplets. These elimination processes are, however, of little importance. The most important removal process for glyoxal in the troposphere is photolysis. [zhu et al., 1996 ] Photolysis of glyoxal can occur through the following pathways [zhu et al., 1996]:

$$
\begin{aligned}
& (\mathrm{CHO})_{2}+h V \rightarrow 2 \mathrm{HCO} \\
& (\mathrm{CHO})_{2}+h V \rightarrow \mathrm{H}+\mathrm{CO}+\mathrm{HCO} \\
& (\mathrm{CHO})_{2}+h V \rightarrow \mathrm{H}_{2}+2 \mathrm{CO} \\
& (\mathrm{CHO})_{2}+h V \rightarrow \mathrm{H}_{2} \mathrm{CO}+\mathrm{CO}
\end{aligned}
$$

Glyoxal photolysis quantum yields, especially radical yields, are of substantial atmospheric interest since free radicals such as HCO (formyl radicals) generated from the photolysis process are readily converted in the atmosphere into $\mathrm{HO}_{2}$ and $\mathrm{OH}$ radicals, i.e.:

$$
\begin{aligned}
& \mathrm{HCO}+\mathrm{O}_{2} \rightarrow \mathrm{CO}+\mathrm{HO}_{2} \\
& \mathrm{HO}_{2}+\mathrm{NO} \rightarrow \mathrm{OH}+\mathrm{NO}_{2}
\end{aligned}
$$

These reactions can ultimately speed up photochemical transformations. 
Molecular orbital energy level diagram

The most characteristic electronic features of the glyoxal molecule are the free oxygen non-bonding ( $n$ ) orbitals and the $\pi$-orbitals on the $\mathrm{C}=0$ groups. To first order, the electronic properties of glyoxal follow from a consideration of the $n, \pi$ one-electron LCAO MOs.

In Figure 1.4, the $\mathrm{n}, \pi$ one-electron orbitals are drawn with their respective symmetry in the $\mathrm{C}_{2} \mathrm{~h}$ point-group (the trans conformer of glyoxal). [Drent and Kommandeur, 1972] Although glyoxal can also be in the cis conformation, it is not as prominent.

To zeroth order, the n-orbitals on the different oxygen atoms are of the same energy thus the orbitals can be represented by a symmetric linear combination $\left(a_{g}\right)$ and an antisymmetric linear combination $\left(b_{u}\right)$. Through interaction with each other and with the $\sigma, \sigma^{*}$-orbitals the energies of these orbitals will split making the antisymmetric linear combination $b_{u}$ lesser in energy. The $\pi$-orbitals are also split in this case by the conjugation across the central cc bond. Upon excitation of the molecule, the $\pi_{3}^{*} \leftarrow n^{+}$and $\pi^{*}{ }_{4} \leftarrow \mathrm{n}^{-}$configurations are electric-dipole allowed whereas $\pi_{4}^{*} \leftarrow n^{+}$and $\pi_{3}^{*} \leftarrow n^{-}$excitations are dipole forbidden by symmetry. [Drent and Kommandeur, 1972] 


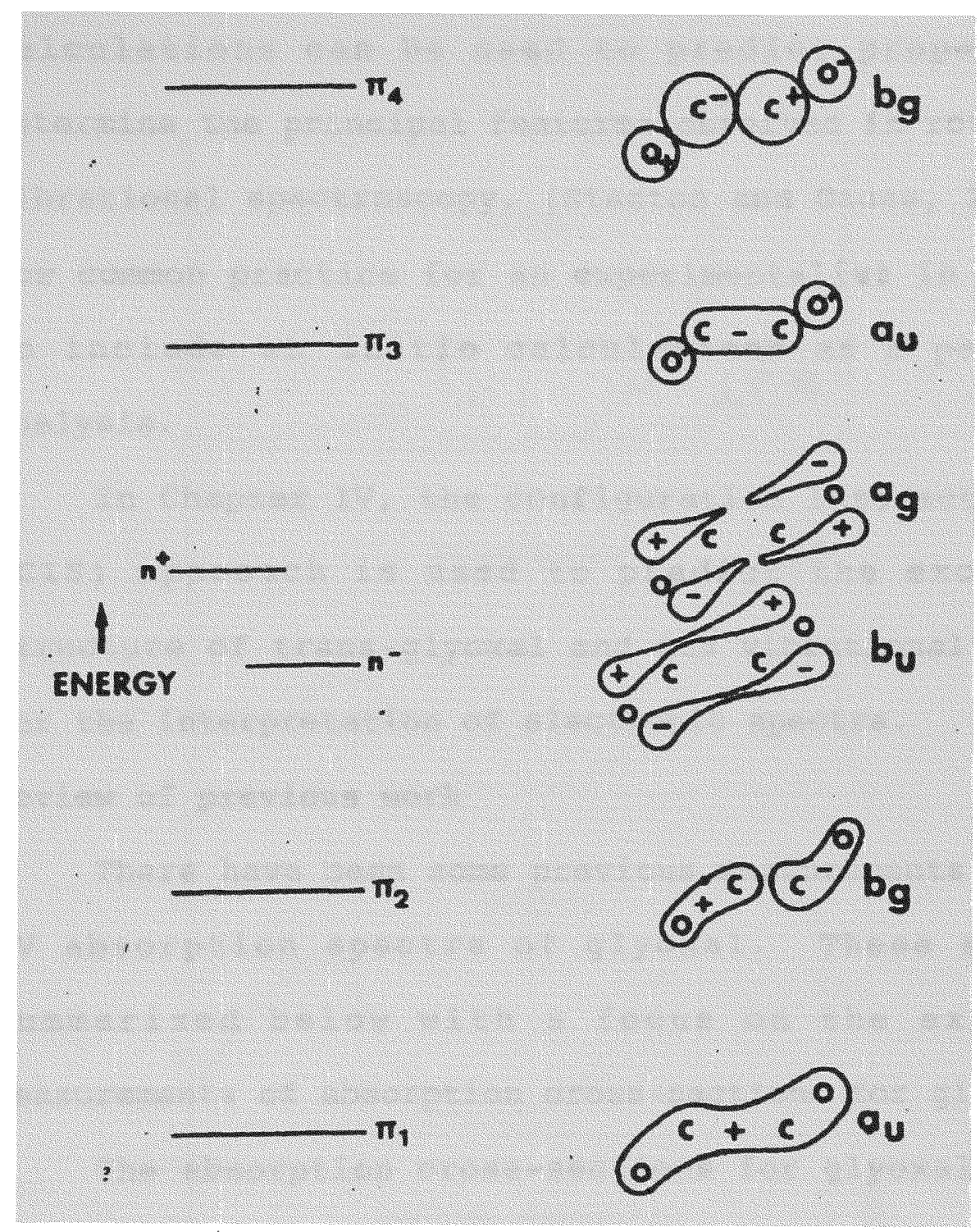

Figure 1.4. Molecular orbitals for trans-glyoxal. [Drent and Kommandeur, 1972]

It is widely recognized that $a b$ initio calculations can be useful in confirming or guiding in assignments in many branches of molecular spectroscopy. When adequate basis sets and correlation treatments are used, such 
calculations can be used to predict properties that determine the principal features observed in rotational and vibrational spectroscopy. [Stanton and Gauss, 1996] It is now common practice for an experimentalist in these areas to include $a b$ initio calculations as a part of data analysis.

In Chapter IV, the configuration interaction singles (CIS) approach is used to predict the excited state structure of trans-glyoxal and its vibrational frequencies for the interpretation of electronic spectra.

\section{Review of previous work}

There have been some previous measurements of the near UV absorption spectra of glyoxal. These studies are summarized below with a focus on the experimental measurements of absorption cross-sections for glyoxal.

The absorption cross-sections for glyoxal by Plum et al. [1983] were determined by using a Cary 17-D spectrophotometer and known pressures ( $-3-13$ torr measured with an MKS Baratron capacitance manometer). Their published gas-phase glyoxal absorption spectra can be seen in Figure 1.5 .

Zhu and coworkers employed excimer laser photolysis for their cross-section measurements. The absorption cross-section of glyoxal at a given photolysis wavelength 


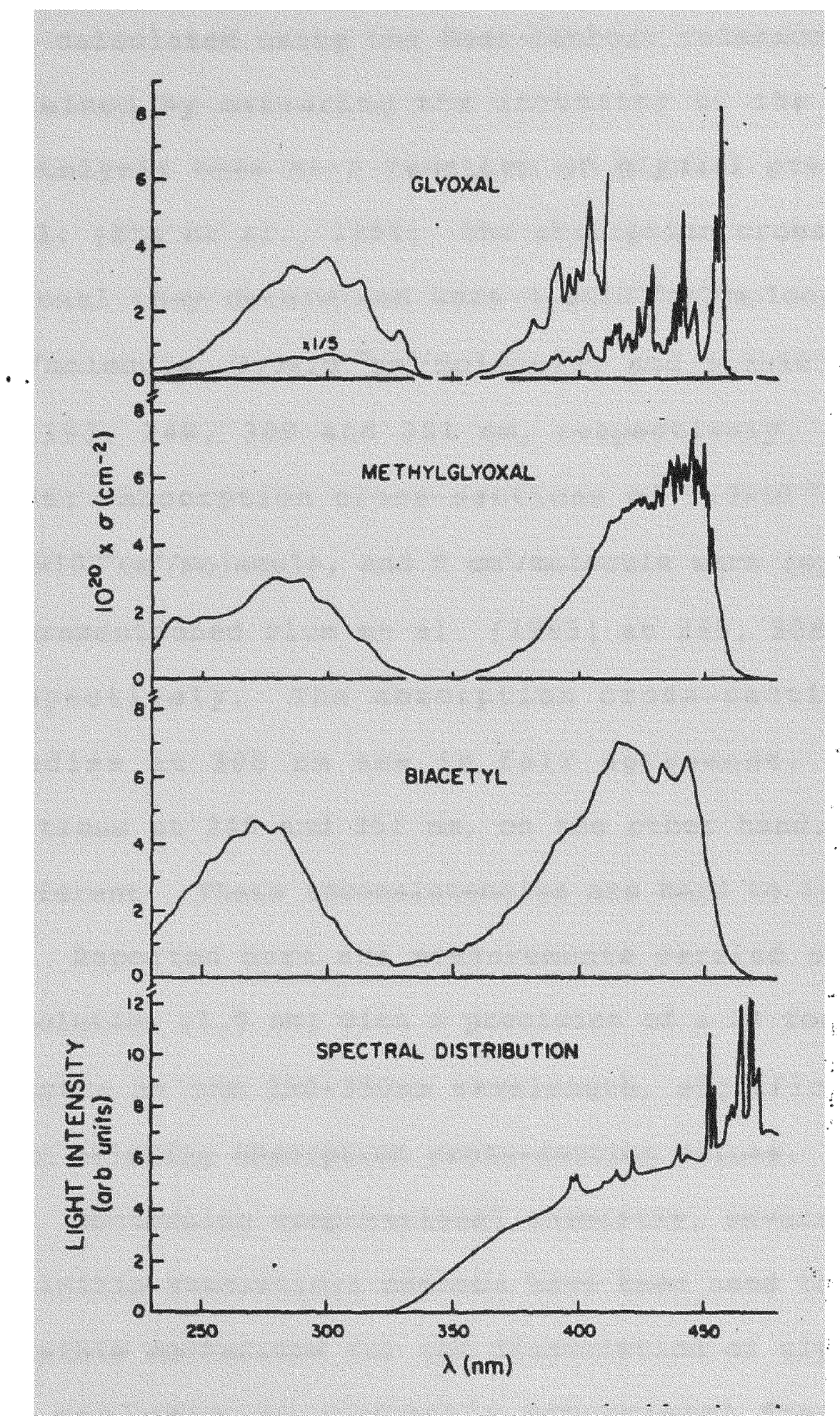

Figure 1.5. (Top) Gas-phase absorption spectra determined for glyoxal by Plum et al., 1983. 
was calculated using the Beer-Lambert relation on the data obtained by measuring the intensity of the transmitted photolysis beam as a function of glyoxal pressure in the cell. [ hhu et al., 1996] The absorption cross-sections of glyoxal they determined were $4.8 \times 10^{-19} \mathrm{~cm}^{2} / \mathrm{molecule}, 1.3 \times 10^{-20}$ $\mathrm{cm}^{2} /$ molecule, $2.9 \times 10^{-20} \mathrm{~cm}^{2} /$ molecule, and $5.1 \times 10^{-21} \mathrm{~cm}^{2} /$ molecule at $193,248,308$ and $351 \mathrm{~nm}$, respectively. [ 2 hu et al., 1996] Absorption cross-sections of $7.3 \times 10^{-21} \mathrm{~cm}^{2} /$ molecule, $2.8 \times 10^{-20} \mathrm{~cm}^{2} /$ molecule, and $0 \mathrm{~cm}^{2} /$ molecule were reported by the aforementioned Plum et al. [1983] at 248, 308 and $351 \mathrm{~nm}$, respectively. The absorption cross-sections of both studies at $308 \mathrm{~nm}$ are in fair agreement. The crosssections at 248 and $351 \mathrm{~nm}$, on the other hand, are largely different. These inconsistencies are hard to ignore.

Reported here are measurements carried out at medium resolution $(1.0 \mathrm{~nm})$ with a precision of $\pm 2 \%$ for the glyoxal spectra at the 200-350nm wavelength, significantly better than existing absorption cross-section values.

Concerning computational chemistry, several high-level $a b$ initio theoretical methods have been used to investigate possible mechanisms for the dissociation of glyoxal as well as analysis of glyoxal's vibrational frequencies and electronic transitions for the ground and excited states. A theoretical study of particular interest is the analysis 
of the structure and harmonic frequencies of planar conformers of glyoxal in their first excited singlet state by Stanton and Gauss [1996]. Their results using a high level of theory will be reviewed in chapter IV.

Alternatively, a different level of theory (CIS approach) was used for this thesis. The computational results were instrumental in the investigation, and ultimately the interpretation of, the spectral region of study 


\section{Experimental data analysis}

\section{Preparation of glyoxal}

Glyoxal is an unstable compound that needs to be either freshly prepared or stored at temperatures of $-78^{\circ} \mathrm{C}$ before use. Some early reports of the production of glyoxal included the oxidation of ethylene with selenium dioxide. [Clavert and Lane, 1953; Carpenter and Foster, 1958] More recent, conventional methods use a derivative precursor of the compound, such as paraglyoxal or glyoxal trimeric hidydrate, as starting materials to obtain glyoxal. Reported below are the two methods that were ultimately attempted.

Plum et. al. [1983] prepared glyoxal by first evaporating a commercially available glyoxal-water solution to dryness under vacuum. Then, after adding and mixing $\mathrm{P}_{2} \mathrm{O}_{5}$ to the resulting polymer-like material, the $\alpha$-dicarbonyl was distilled off under vacuum and collected at $77^{\circ} \mathrm{K}$. [Plum et al. 1983]

Zhu et al. [1996], on the other hand, produced glyoxal by heating glyoxal trimeric dihydrate in the presence of $\mathrm{P}_{2} \mathrm{O}_{5}$. [Langford and Moore, 1984] The glyoxal samples were 
purified by repeated freeze-pump-thaw cycles and were stored at liquid-nitrogen temperature before use.

The first attempt to prepare glyoxal was based on the method used by Plum and coworkers. A commercially available aqueous solution that was $40 \%$ glyoxal [Aldrich] by mass was evaporated to dryness under vacuum using the main manifold. (See Figure 2:1) After drying a paraglyoxal polymer was observed. The polymer was under vacuum and stored in the refrigerator. Two days later the distillation of glyoxal was attempted. A manifold with six valves similar to the one shown in Figure 2.1 was used for the distillation. The paraglyoxal was placed on the far left-hand side and a trap on the far right hand side. $\mathrm{P}_{2} \mathrm{O}_{5}$ (phosphorus pentoxide) was mixed with the paraglyoxal before heating. The vacuum pump was placed in the middle value. A water bath was used to heat the paraglyoxal. The bath water reached a temperature of $90^{\circ} \mathrm{C}$. There seemed to be some initial vaporization of the polymer going on as the temperature increased but then a point was reached where no additional distillation occurred. No useful amounts of glyoxal were obtained by this procedure.

In the next attempt $15 \mathrm{ml}$ of the glyoxal-40\% water solution was placed in a round bottom flask and the water pumped off on the main manifold. A rubber stopper was 


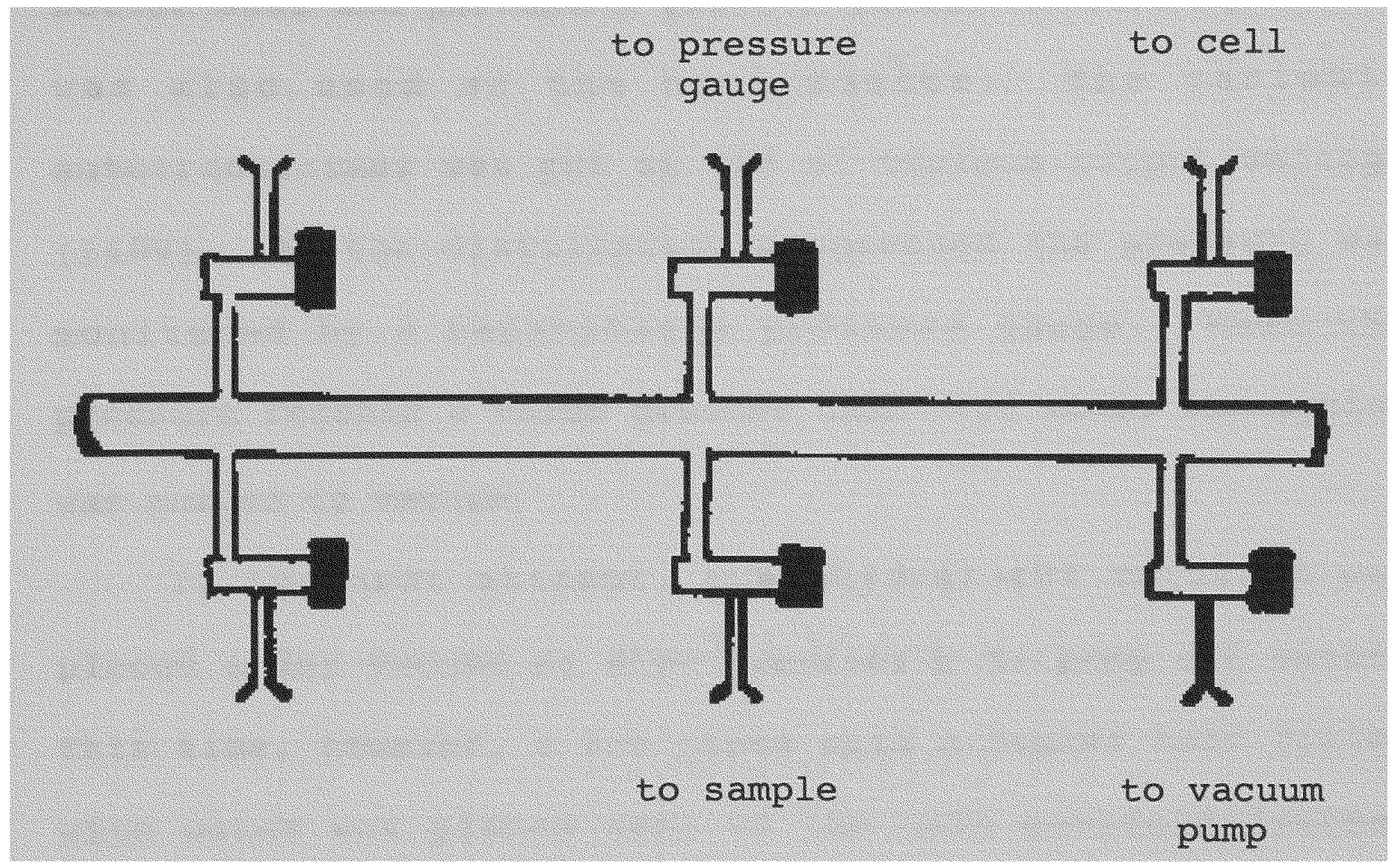

Figure 2.1. Schematic diagram of the gas handling manifold.

joined to a glass connector for the vacuum system and used to stopper the round bottom flask. A few days later, the contents of the round bottom flask were distilled under the hood using the five-valve manifold. Before connecting the round bottom flask an excess of $\mathrm{P}_{2} \mathrm{O}_{5}$ was mixed into the round bottom flask. During the distillation yellow prisms were seen in the cell (in the trap) consistent with production of glyoxal. It was once again, however, not possible to obtain useful quantities of the compound.

The preparation was attempted again with a new glass stopper, replacing the rubber stopper. This was to give a 
better seal and perhaps a greater yield. A heating mantle was also used as the heat source. The variable autotransformer was put at $40 \%$ of maximum output voltage $(140 \mathrm{~V})$. As the distillation progressed the pressure was monitered by a capacitance pressure gauge. When the pressure reached a value greater than $\sim 400$ torr, the system was pumped to vacuum.

In the next attempt, the glyoxal-40\% solution was placed under vacuum as done previously to pump off water. This time, however, a hot plate with a beaker half filled with water was placed next to the main manifold system. After pumping off the water for hours, the round bottom flask was submerged in the beaker with hot water to ensure as much of the water was pumped off as possible. The paraglyoxal looked different from the previous times. Its appearance was like that of an airy polymer. The crystals formed were white flat crystals with a "naphthalene" like appearance. No phosphorus pentoxide was put into the round bottom flask for the distillation. After being distilled, there seemed to be hot spots created from the heating mantle on the round bottom flask. The contents of the flask appeared to be much browner on one small section of the flask than the rest. The yellow vapor reached almost to the second valve of the manifold set up but not any 
further. The cell in the trap was checked frequently but the yellow crystals seen in the first attempt was never seen. The residue left behind in the round bottom flask appeared to be just the burnt glyoxal.

Because of the lack of success in obtaining glyoxal from the aqueous solution, the next attempt to prepare glyoxal was based on the method of $\mathrm{zhu}$ and coworkers. Five grams of glyoxal trimeric dihydrate [Aldrich] and 2.5 grams of phosphorus pentoxide were placed in the round bottom flask. A different distillation apparatus was also set up. (See Figure 2.2) The flask was heated for approximately 40 minutes (variable autotransformer at $40 \%$ ) when a pale yellow crystalline product was observed in the collection round bottom flask.

Based on the apparent success of this procedure the method was carried out a second time using fifteen grams of the glyoxal trimeric dihydrate along with seven grams of phosphorus pentoxide. A greater yield was obtained. An IR cell was filled with the product and a spectrum was taken. (See Figure 2.3) The peak intensities and locations for glyoxal were compared to the experimentally known values. The spectrum of the product was consequently determined to be glyoxal. The same cell was also filled with benzene, used as a reference spectrum. (See Figure 2.4) 


\section{Apparatus}

Absorption measurements were made on a shimadzu UV2101 PC (Zenith Data system) scanning spectrophotometer. The spectrophotometer is a double beam instrument capable of obtaining spectra in the wavelength region $190-800 \mathrm{~nm}$, at a resolution of $0.1 \mathrm{~nm}$ in the UV-visible spectral region. The sample compartment of the spectrophotometer was modified to fit a cylindrical gas absorption cell.

The gas absorption cell was constructed by attaching a pair of one-inch diameter supracil quartz windows to both ends of a $10 \mathrm{~cm}$ length, $25 \mathrm{~mm}$ diameter glass tube. A glass stopcock was attached to the body of the cell to allow filling of the cell on a vacuum manifold.

Other glassware, such the gas handling manifolds and the distillation set up, were thoroughly cleaned with nitric acid, a soap solution, water, and deionized water, and then dried before use.

A diagram of the experimental apparatus is given in Figure 2.5.

\section{Test of the apparatus}

To check that the instrument is functioning correctly, the Shimadzu UV 2101 spectrophotometer has a number of self-testing sequences that are run automatically at startup. Due to the nature of the experiments, however, 


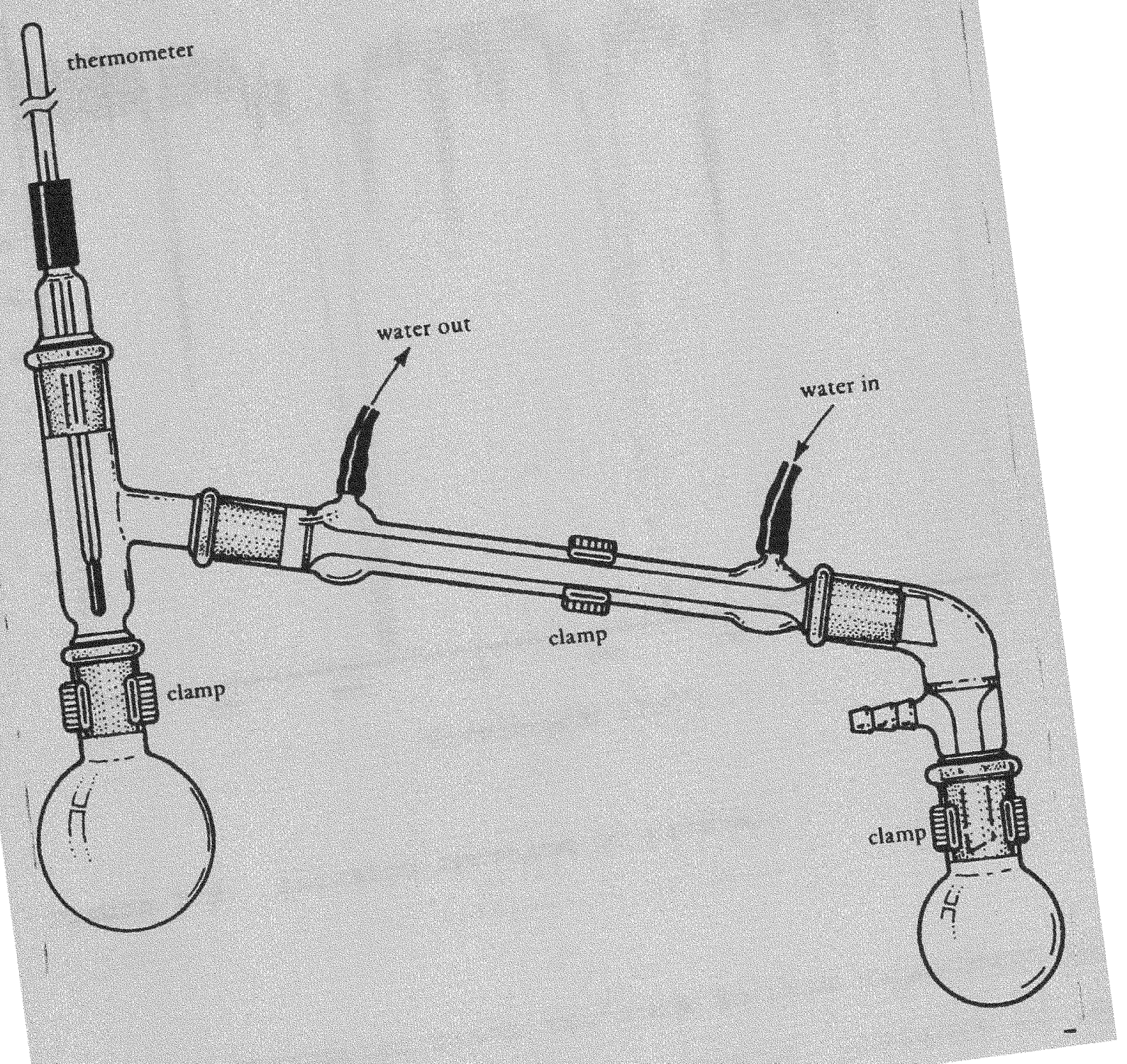

Figure 2.2. Distillation apparatus.

aditional tests of the instrument were carried as discussed below. 


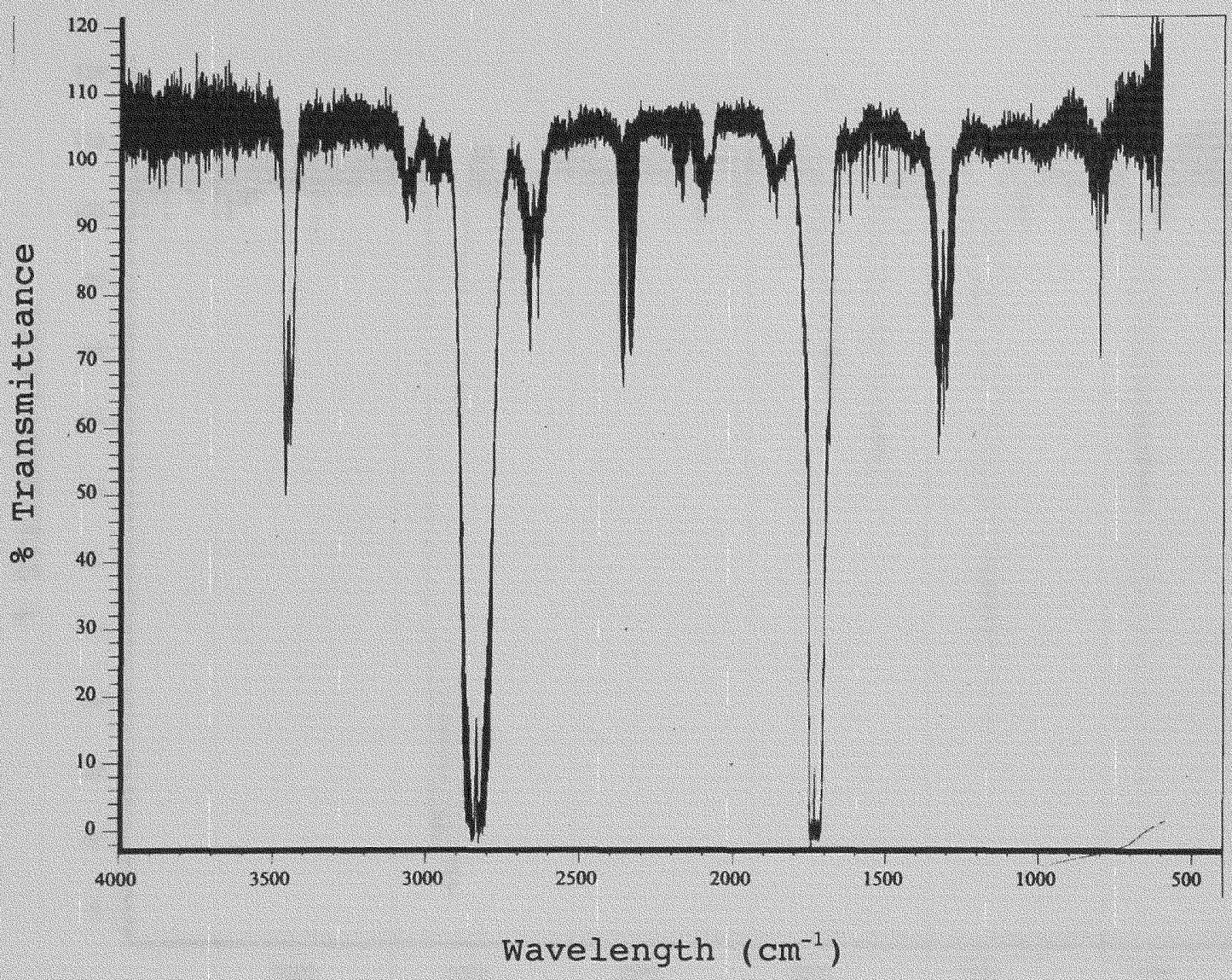

Figure 2.3. Infrared spectrum of glyoxal.

The absorption cross sections in this experiment are obtained from a plot of absorbance vs. pressure of the absorbing gas. Absorption linearity was tested by plotting absorbance vs. concentration for a series of potassium dichromate solutions. A stock solution of approximately $200 \mathrm{mg} / 1$ potassium dichromate was prepared and diluted to volume with a $0.05 \mathrm{M}$ aqueous potassium hydroxide solution. Serial dilutions of this stock solution were prepared with 


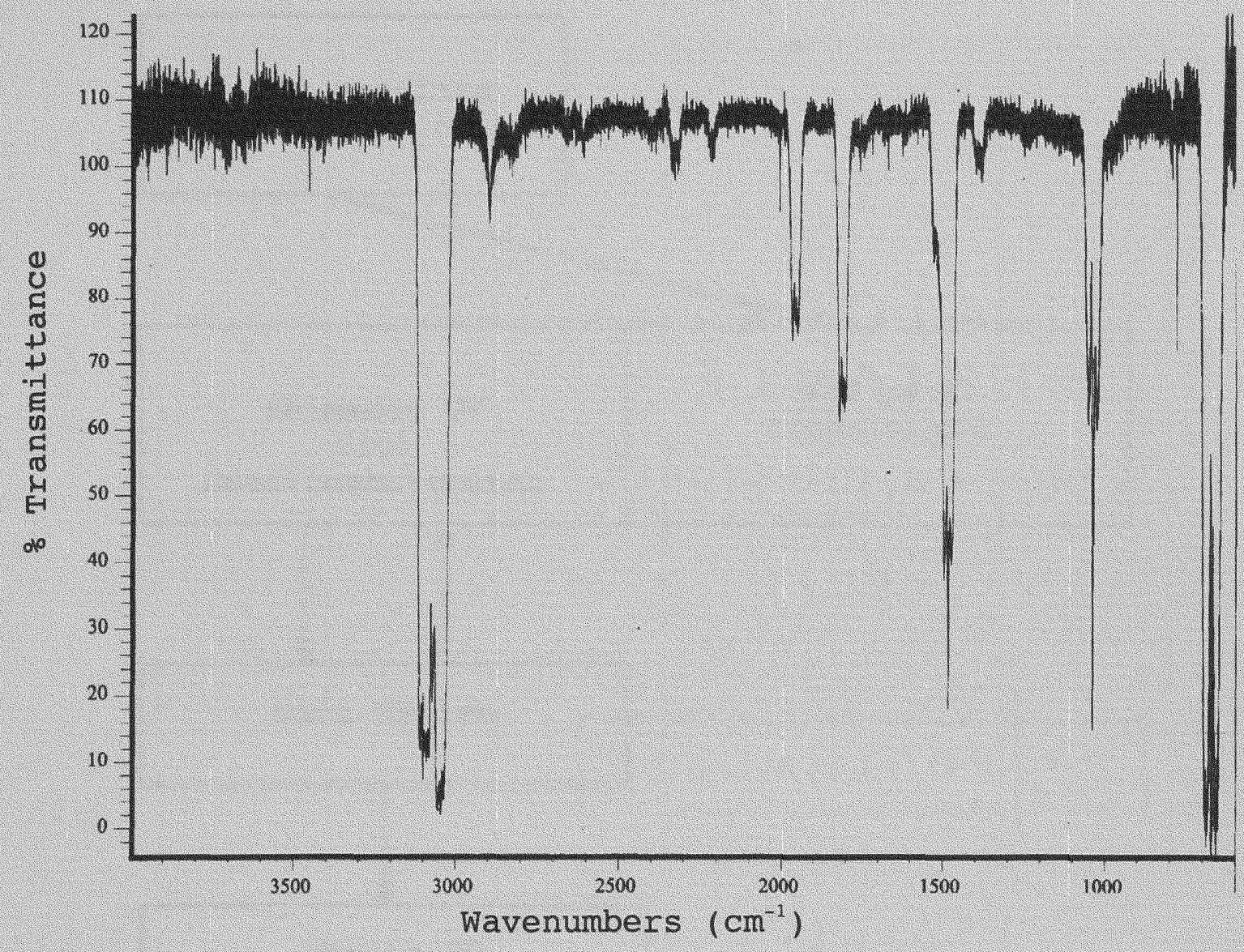

Figure 2.4. Infrared spectrum of benzene.

concentrations from 10-50 mg/l. Absorbance was measured at $320 \mathrm{~nm}$ and a spectral bandwidth of $1.0 \mathrm{~nm}$. A plot of these intercept, and correlation coefficient for the data showed that no deviations from Beer's law occurred for absorbances in the range of $0-2.5$ absorption units.

Wavelength calibration

Two methods were performed for checking the wavelength 


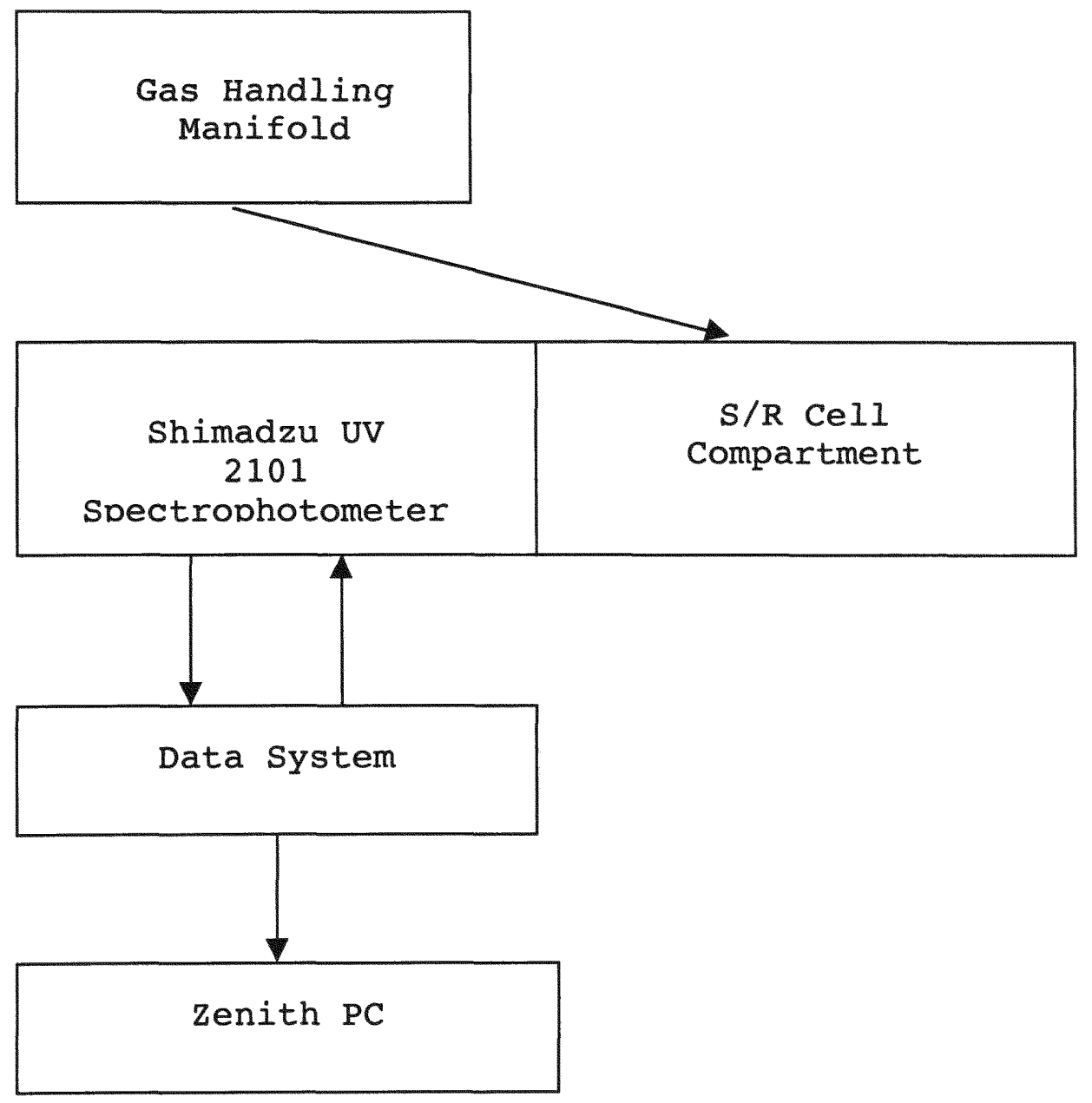

Figure 2.5. Schematic diagram of the experimental apparatus.

calibration of the spectrophotometer. They were instrument self check and the measurement of benzene vapor spectrum. In the instrument self test, the Shimadzu UV 2101 spectrophotometer uses the emission lines of the deuterium 


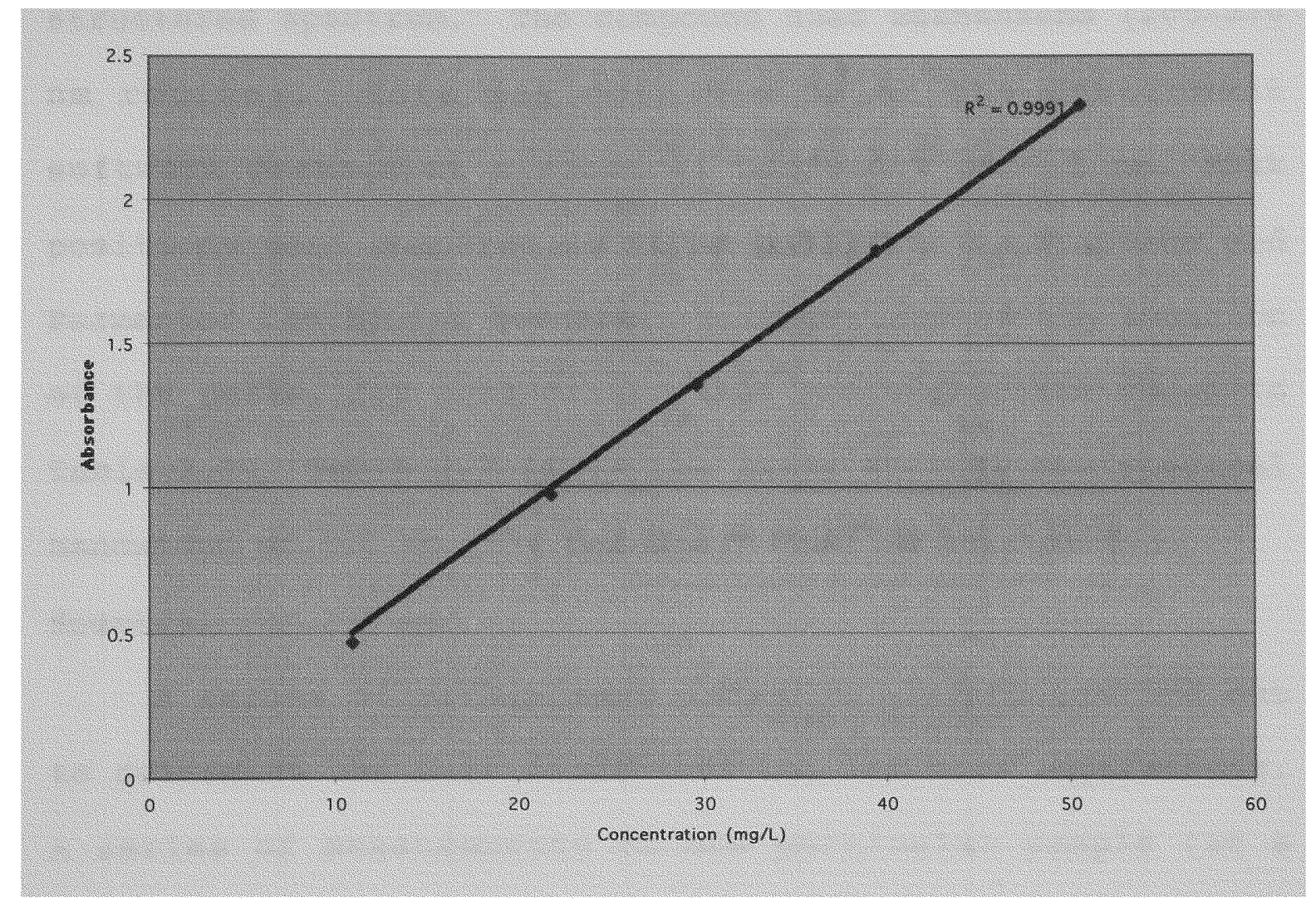

Figure 2.6. Absorbance vs. concentration for the potassium dichromate calibration solutions.

lamps, or overtones of these lines, whose wavelengths are known. The measured values for wavelength are compared with the known values. When turning on the instrument, any unacceptable deviation from these values results in the instrument requesting troubleshooting.

A second calibration test was carried out by measuring the vapor phase spectrum of a compound possessing a highly 
structured spectrum. The compound used wasbenzene (200-270 nm region). Data was obtained using the instrument software package at a spectral bandwidth of $0.2 \mathrm{~nm}$. Peak positions were compared to those published by Atkinson and Parmenter [1978] for benzene. A comparison of the location of the peaks, for this calibration procedure, are shown in Table 2.1. Table 2.2 lists the peaks seen at the spectral bandwidth of $1.0 \mathrm{~nm}$ (the parameter used in this study).

\section{Spectral resolution}

A series of preliminary experiments were carried out to determine the best conditions for the main experiments. A series of measurements on one particular sample (at a pressure of 100 Torr) were performed. In these measurements the sample was viewed at different values of spectral resolution and instrument response time. It was decided, based on the results, that the best spectral resolution for the measurements was $1.0 \mathrm{~nm}$. This is appropriate for an absorption spectrum containing no sharp structured features. The relationship in the determining scan rate is:

Scan rate < Band width/response time.

Based on this relationship a scan rate of $0.91 \mathrm{~nm} / \mathrm{sec}$ was 
Comparison of Benzene Peak Location

\begin{tabular}{|c|c|c|}
\hline Peaks & Location $^{a}\left(\mathrm{~cm}^{-1}\right)$ & Location $^{\mathrm{b}}\left(\mathrm{cm}^{-1}\right)$ \\
\hline 1 & 37481.6 & 37453.2 \\
\hline 2 & 38522.5 & 38432.0 \\
\hline 3 & 38611.3 & 38580.2 \\
\hline 4 & 39038.5 & 39062.5 \\
\hline 5 & 39534.6 & 39494.5 \\
\hline 6 & 39638.0 & 39745.6 \\
\hline 7 & 40131.9 & 40160.6 \\
\hline 8 & 40456.7 & 40420.4 \\
\hline 9 & 40968.5 & 40916.5 \\
\hline 10 & 41165.3 & 41186.2 \\
\hline 11 & 41254.1 & 41254.1 \\
\hline 12 & 41478.6 & 41356.5 \\
\hline 13 & 41924.7 & 42052.1 \\
\hline 14 & 42298.0 & 42265.4 \\
\hline 15 & 43009.3 & 42992.3 \\
\hline 16 & 43215.7 & 43177.9 \\
\hline
\end{tabular}

(a)From Atkinson and Parmenter [1978]

(b) Shimadzu instrument

Table 2.2

Location of Benzene Peaks at $0.1 \mathrm{~nm}$ Bandwidth (Parameter Used in Study)

Peak

1

5

7

12

13

15
Location $\left(\mathrm{cm}^{-1}\right)$

37453.2

39447.7

40322.6

41322.2

42194.1

42918.5 
selected. A number of different sampling intervals were also used. The spectrum could be satisfactorily reproduced using a spacing of $0.5 \mathrm{~nm}$ between adjacent data points.

\section{Data acquisition and analysis}

The purpose of the experiments was to obtain accurate room temperature absorption cross sections for glyoxal at the $280 \mathrm{~nm}$ wavelength. The steps involved in the data acquisition, transfer, and analysis procedure are outlined in Table 2.3. This involved measurement of experimental absorbances as a function of wavelength and pressure of absorbing gas, and conversion of this data into absorption cross section values.

Table 2.3

Summary of the Data Analysis Process

Step

Measure spectrum

Transfer data to Zenith P.C.

Convert data to absorbances

Align data

Combine data into a single file

Calculate absorption cross-sections

Combine cross-sections into a single file
Program

SPECDATA.BAS

DATATRAN . BAS

DATARED.BAS

PLOTDATA. BAS

PLOTSUM. BAS

DATAANAL. BAS

SUMMARY.BAS 
The experimental data was obtained on a Shimadzu UV 2101 scanning spectrophotometer interfaced to a PC (zenith Data system). The experimental conditions (i.e. wavelength range and other operating conditions) are given in Table 2.4. All spectra were measured at room temperature $\left(-20^{\circ} \mathrm{C}\right)$ at a spectral bandwidth of $1.0 \mathrm{~mm}$, a scan rate of 0.91 $\mathrm{nm} / \mathrm{sec}$, and a sampling interval of $0.5 \mathrm{~nm} /$ data point.

\section{Table 2.4}

Operating conditions for the spectrophotometer

Spectral Bandwidth

Scan Rate

Spacing between data points
$1.0 \mathrm{~nm}$

$0.91 \mathrm{~nm} / \mathrm{sec}$

$0.5 \mathrm{~nm}$

Experimental data was obtained by first running a baseline spectrum with the empty reference gas absorption cell in place, then measuring the absorption spectrum over the particular wavelength range in the following sequence:
B1 = Empty sample cell with a pressure of 0 (2.2a) Torr

$\mathrm{S1}, \mathrm{s} 2=$ cell filled with absorbing gas at $(2.2 \mathrm{~b})$ 


\section{a known pressure of glyoxal \\ B2 = Empty sample cell with a pressure of 0 (2.2c) Torr}

A spectrum was measured with the empty sample cell. The cell was then filled with glyoxal at a known pressure and measured. Duplicate measurements were made of the filled cell. The cell was then evacuated and a second blank spectrum was run.

The sequence of four spectra (B1, S1, S2, B2) for the particular wavelength range and an absorbing gas pressure were stored as a sequence of data point pairs containing the experimental wavelength and absorbance. The data for each spectrum were temporarily stored on diskette in four separate files, named BXYYY for the blanks. $X$ was assigned based on the number of samples run that day and varied from one to five. The YYY stood for the first letter of the month the spectra was run followed by the day. The samples were named FX1YYY and FX2YYY, where X depended on the sequence that the sample was run that day and the YYY for the samples were labeled following the same format as the blanks. The actual acquisition of data was done following the sequence in Equations $2.2 a, b, c$. Date analysis 
The next step in the data analysis consisted of taking the four experimental files (B1, S1, S2, B2) and converting them into a single file of experimental wavelengths and absorbances. This was accomplished using the program DATARED.BAS, a data reduction program.

For each wavelength an experimental absorbance was calculated using the relationship:

$$
A=[(S 1+S 2)-(B 1+B 2)] / 2
$$

where $A$ is the experimental absorbance. By subtracting the blank spectrum ( $\mathrm{B} 1+\mathrm{B} 2)$ from the sample spectrum $(\mathrm{S} 1+\mathrm{S} 2)$, a correction was made for a possible drift in the instrument baseline. The result of the above process was a reduced data set of absorbances.

Absorbance/pressure vs. wavelength were plotted using PLOTDATA.BAS for a series of runs. Those runs that showed evidence of degradation or baseline drift were discarded. Once a set of reliable experimental data had been obtained cross-section calculations were initiated.

\section{Cross-section calculation}

The absorption cross-section for glyoxal could be calculated. This was accomplished by using the method of linear least squares to find the best fitting line to the 
data. The absorption cross-section, $\sigma$ (in units of $\mathrm{cm}^{2}-$ molecule $e^{-1}$ ) is given by the equation;

$$
\sigma=(2.303 / 1)(\mathrm{dA} / \mathrm{dN})
$$

where 1 is the path length of the cell $(9.803 \mathrm{~cm}) . \mathrm{dA} / \mathrm{dN}$ is related to $\mathrm{dA} / \mathrm{dP}$, the slope of the best fitting line in the plot of absorbance vs. pressure, by the relationship:

$$
\mathrm{dA} / \mathrm{dP}=\left(1.379 \times 10^{-3} \text { molecule/torr }-\mathrm{Cm}^{3}\right)(\mathrm{dA} / \mathrm{dN})
$$

where the conversion factor between $d A / d P$ and $d A / d N$ is calculated assuming all pressures are reported at $\mathrm{T}=298.15^{\circ}$ $\mathrm{K}$.

The data for individual wavelengths can be plotted to check the reliability of the data. An example set of the data is shown in Figure 2.7. The absorption cross-section $\sigma$, calculated by the above procedure, is the coefficient seen when Beer's law is written in the form:

$$
I / I_{0}=\exp (-\sigma N 1)
$$

where $I_{0}$ is the initial intensity, I is the light intensity after passing through the cell, $\sigma$ is the absorption cross- 


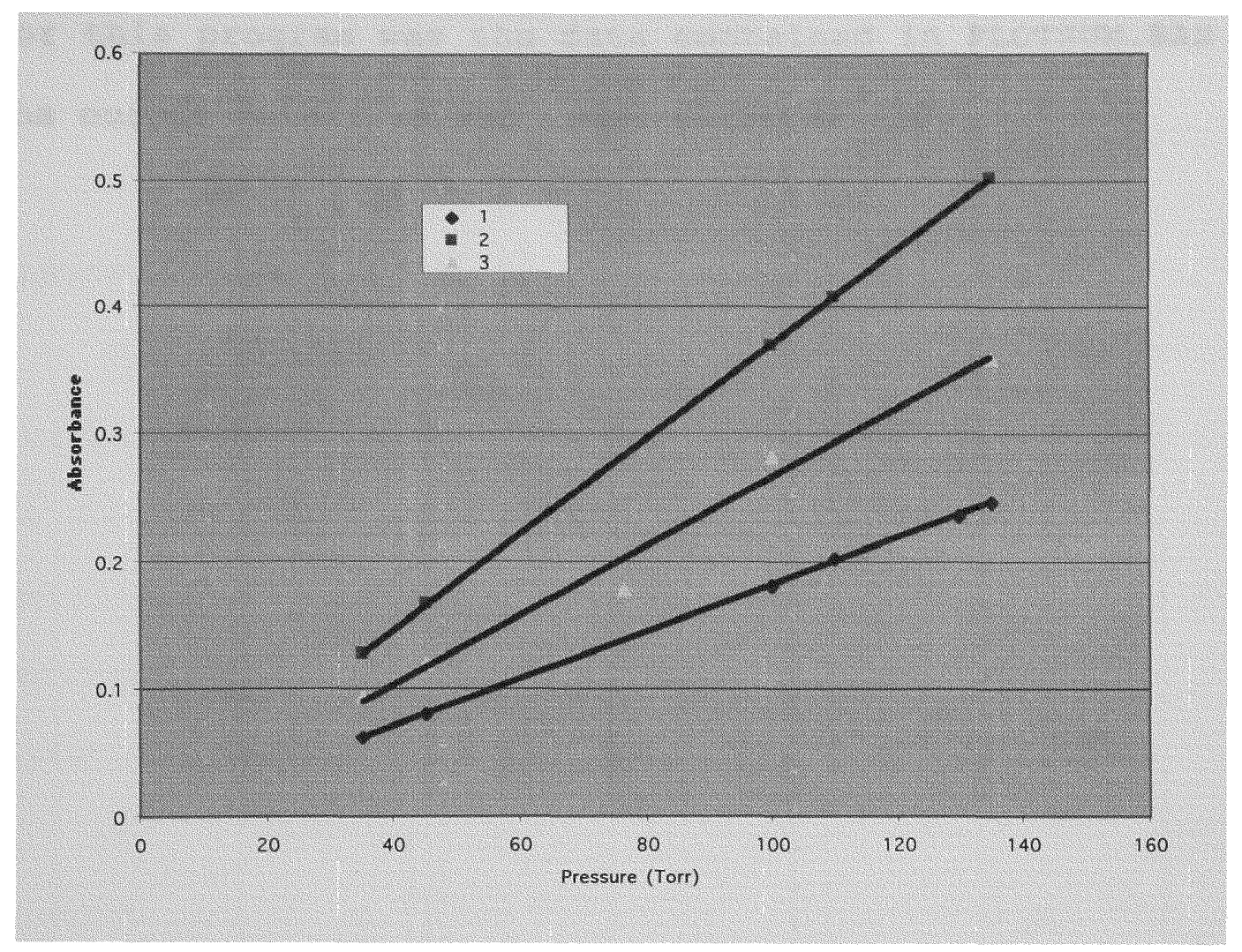

Figure 2.7. Absorbance vs. pressure for glyoxal at (1) $330 \mathrm{~nm}$, (2) $310 \mathrm{~nm}$, and (3) $270 \mathrm{~nm}$.

section discussed earlier, $\mathrm{N}$ is the number density of absorbing gas molecules in units of molecules $-\mathrm{cm}^{-3}$, and 1 is the path length in $\mathrm{cm}$.

The calculations of the absorption cross-sections were carried out in the program DATAANAL.BAS. The input data 
for this program was the data contained in PLOTSUM.BAS and the output data file was named GLYSHORT.ASC. 


\section{Chapter III}

\section{Experimental Results}

\section{Summary of results}

The final results for the absorption cross-sections for glyoxal, obtained from the experimental data, are shown in Figure 3.1. The complete set of absorption crosssection values as a function of wavelength is presented in Appendix A.

\section{Comparison with previous works}

The results from the measurements of the near UV absorption spectra of glyoxal are represented as absorption cross-sections. The cross-sections are given at a spacing interval of $0.5 \mathrm{~nm}$ and at a temperature of $298.15 \mathrm{~K}$. The range of wavelengths for which the values are reported is 200-350 nm. Table 3.1 shows a comparison between the results obtained in this study, Plum et al. [1983], and zhu et al. [1996].

The glyoxal absorption cross-sections at $308 \mathrm{~nm}$ are all in good agreement. The cross-section at $248 \mathrm{~nm}$ reported by zhu et al. [1996] is larger than those determined by both this study and Plum et al. [1983] - The values reported in this thesis, and those found by Plum and coworkers, however, are very similar. 


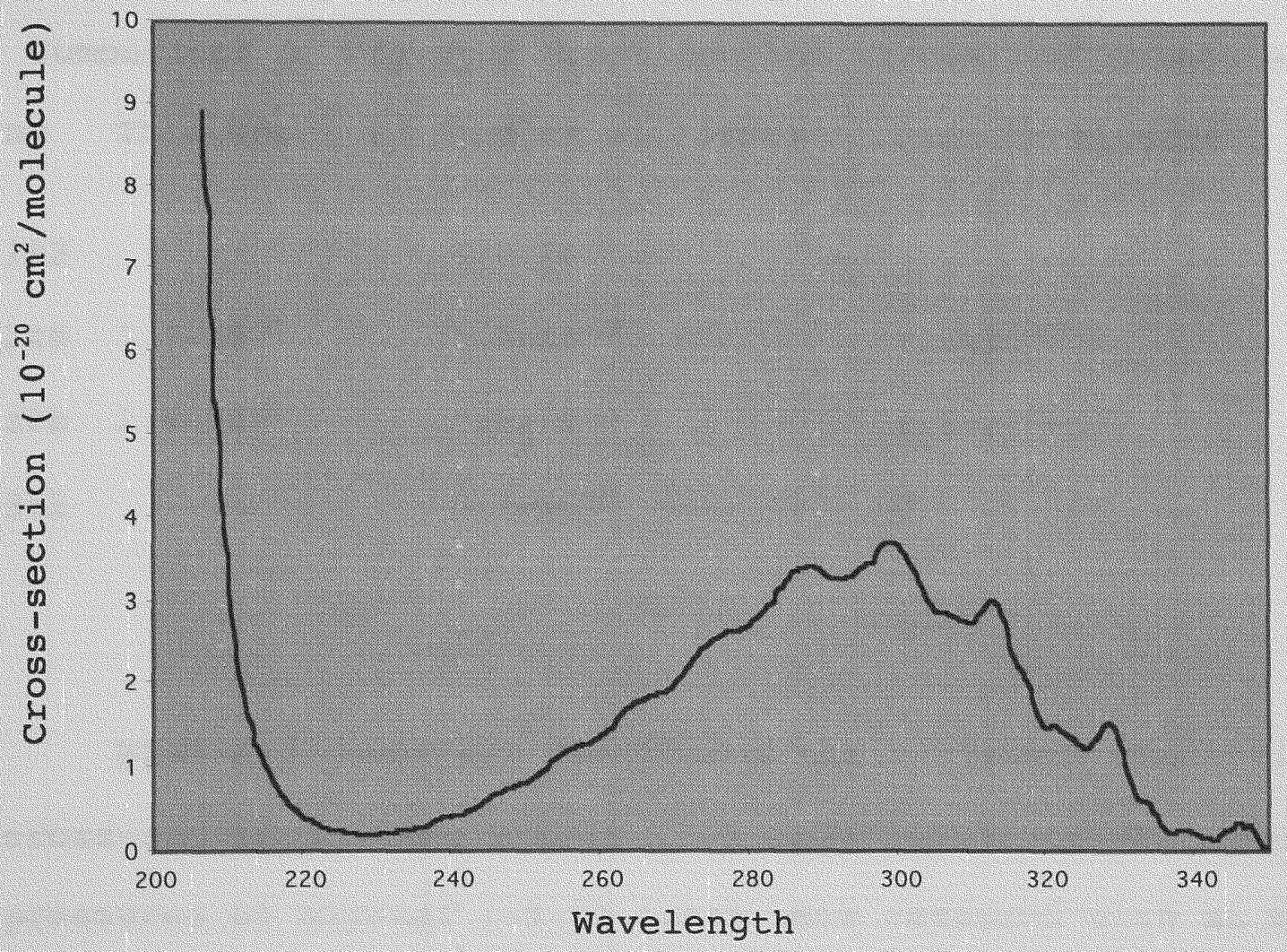

Figure 3.1. Spectra of glyoxal.

The wavelength region in this study, that is 200-350 nm, did not include the other two wavelengths listed. However, it should be mentioned that at the $351 \mathrm{~nm}$ wavelength, zhu et al. [1996] reports a glyoxal crosssection significantly larger than Plum et al. [1983]. 


\section{Table 3.1}

Comparison of Previous Cross-Section Values $\left(\mathrm{cm}^{2} / \mathrm{molecule}\right)$

nm This Work

$$
\text { Zhu et al.(1996) Plum et al.(1983) }
$$

193

$$
4.8 \times 10^{-19}
$$

$248 \quad 7.7 \times 10^{-21}$

$1.3 \times 10^{-20}$

$7.3 \times 10^{-21}$

$3082.8 \times 10^{-20}$

$2.9 \times 10^{-20}$

$2.8 \times 10^{-20}$

351

$5.1 \times 10^{-21}$

0

Plum and coworkers determined their glyoxal absorption cross-sections using a Cary 17-D spectrophotometer. Known pressures of glyoxal ( -3-13 torr) were measured with an MKS Baratron capacitance manometer. The experimental apparatus used in the study for this thesis were similar but the range of pressures of glyoxal are larger, that is $26-135$ torr. Zhu et al. (1996), on the other hand, employed excimer laser photolysis at 193, 248, 308 and $351 \mathrm{~nm}$ in combination with cavity ring-down spectroscopy. zhu et al. (1996) claim they checked the purity of the glyoxal, repeatedly measured its absorption cross-sections at the reported photolysis wavelengths and have confidence in their results. 
As was noted above, however, the results for both studies are similar. Further studies should be conducted to verify what the cross-section measurements are at the wavelengths with conflicting values. 


\section{Chapter IV}

\section{Computational Theoretical Background}

Presently, it is possible to run computational programs in order to obtain information pertaining to molecules. Electronic structure calculations have become a common tool to predict the equilibrium geometry of molecules. In fact, the total energy of a molecule can be defined by a potential energy surface. The points on this potential surface represent solutions to questions that can help in the prediction of chemical structures. For this thesis, calculations were run to explain what is happening to the glyoxal molecule during photodissociation, that is how it breaks apart and the interpretation of the region of study

\section{The Schrodinger Equation}

Using the laws of quantum mechanics, the energy and other related properties of a molecule may be obtain by solving the schrodinger equation [Atkins and Freidman, 1997] :

$$
\mathrm{H} \Psi=\mathrm{E} \Psi
$$


The operator in Equation (4.1) that returns the system energy, E, as an eigenvalue is called the Hamiltonian operator, H. This Hamiltonian operator typically takes into account five contributions to the total energy of a molecule: the kinetic energies of the electrons and nuclei, the attraction of the electrons to the nuclei, and the interelectronic and internuclear repulsions. [Cramer, 2002] Placing the Hamiltonian into mathematical notation:

$$
\begin{aligned}
\mathrm{H}=- & -\sum(\mathrm{h} / 2 \pi)^{2} / 2 \mathrm{~m}_{\mathrm{e}} \nabla_{i}^{2}-\sum(\mathrm{h} / 2 \pi)^{2} / 2 \mathrm{~m}_{\mathrm{k}} \nabla_{\mathrm{k}}^{2}-\sum \sum \mathrm{e}^{2} \mathrm{z}_{\mathrm{k}} / \mathrm{r}_{\mathrm{ik}} \\
& +\sum \mathrm{e}^{2} / \mathrm{r}_{\mathrm{ij}}+\sum \mathrm{e}^{2} \mathrm{z}_{\mathrm{k}} \mathrm{z}_{1} / \mathrm{r}_{\mathrm{k} 1}
\end{aligned}
$$

where $i$ and $j$ run over electrons, $k$ and 1 run over nuclei, $h$ is Planck's constant, $m_{e}$ is the mass of the electron, $m_{k}$ is the mass of nucleus $k, \nabla^{2}$ is the Laplacian operator, e is the charge on the electron, $z$ is an atomic number, and $r_{a b}$ is the distance between particles $a$ and $b$.

\section{The Born-oppenheimer approximation}

The Hamiltonian in Equation (4.2) contains pairwise attraction and repulsion terms implying that no particle is moving independently of all of the others, $i$. e. there is an interdependency. As a result, accurate wavefunctions for such systems are extremely difficult to express because of the correlated motions of particles, and solving the 
Schrodinger equation even for a simple molecule is not an easy task. This is where the Born-Oppenheimer approximation becomes useful. The Born-oppenheimer approximation is based on the fact that, under typical physical conditions, the nuclei of molecular systems are moving much more slowly than the electrons. For practical purposes, the electrons react essentially instantaneously to changes in nuclear position. Thus, the electron distribution within a molecular system depends on the positions of the nuclei, and not on their velocities. [Foresman and Frisch, 1996] Put another way, the electrons can be described as occurring in a field of fixed nuclei.

Hartree-Fock theory

D. R. Hartree introduced a method to calculate the energy and the ground state wavefunction of an atom in 1928. [Hartree, 1928] In this method, the Schrodinger equation is examined in the context of a one-electron Hamiltonian. As such, the only terms in the Hamiltonian are the one-electron kinetic energy and nuclear attaction terms. Moreover, the operator is 'separable' and may be expressed as:

$$
\mathrm{H}=\Sigma \mathrm{h}_{\mathrm{i}}
$$


where $\mathrm{N}$ is the total number of electrons and $h_{i}$ is the oneelectron Hamiltonian defined by;

$$
h_{i}=-1 / 2 \nabla_{i}^{2}-\Sigma z_{k} / r_{i k}
$$

where $M$ is the total number of nuclei. The eigenfunction of the one-electron Hamiltonian defined in the above Equation (4.4) must satisfy the corresponding one-electron Schrodinger equation:

$$
\mathrm{h}_{\mathrm{i}} \psi_{\mathrm{i}}=\varepsilon_{\mathrm{i}} \psi_{\mathrm{i}}
$$

Since the Hamiltonian operator defined by Equation (4.3) is separable, its many-electron eigenfunctions can be constructed as products of one-electron eigenfunctions, i.e.

$$
\Psi_{\mathrm{HP}}=\psi_{1} \psi_{2} \ldots \psi_{\mathrm{N}}
$$

The Hamiltonian defined by Eqs. (4.3) and (4.4) does not include interelectronic repulsion. This computation depends not on one electron, but instead on all possible (simultaneous) pair-wise interactions. The Hartree-product wavefunction $\Psi_{\text {нр }}$ can be useful in computing energies from 
the correct Hamiltonian. That is, the goal is to find orbitals that minimize $\left\langle\Psi_{\mathrm{HP}}|\mathrm{H}| \Psi_{\mathrm{HP}}\right\rangle$. By applying variational calculus, one can show that each such orbital $\psi_{i}$, is an eingenfunction of its own operator $h_{i}$ defined by;

$$
h_{i}=-1 / 2 \nabla_{i}^{2}-\sum z_{k} / r_{i k}+v_{i}\{j\}
$$

where the final term represents an interaction potential with all of the other elections occupying orbitals $\{j\}$ and may be computed as;

$$
v_{i}\{j\}=\sum \int \rho_{j} / r_{i j} d r \quad(4.8)
$$

where $\rho_{j}$ is the charge (probability) density associated with electron j. [Cramer, 2002] The repulsive third term on the right hand side of Equation (4.7) is thus, exactly analogous to the attractive second term, except that the nuclei are treated as point charges, while electrons, being treated as wavefunctions, have their charge spread out, requiring an integration over all space. [Cramer, 2002] In short, the field seen by electron $i$, depends on the orbitals of the other electrons. Thus, the equation is nonlinear and must be solved iteratively. 
Hartree proposed an iterative 'self-consistent field' (SCF) method to finesse this problem. In the first step of the SCF process, one initially guesses the wavefunctions for all of the occupied MOs constructing the necessary oneelectron operators, h. Solution of each differential equation (4.5) provides a new set of wavefunctions. These presumably more accurate wavefunctions are used to form anew the one-electron Hamiltonians and ultimately determine each necessary $\rho$. The process is repeated to obtain a still better set of wavefunctions. At some point, the difference between a newly determined set and the immediately preceeding set falls below some threshold criterion, and the final set of wavefunctions is referred to as the 'converged' SCF orbitals.

One problem with the Hartree wavefunction is that they do not involve spin and are not anti-symmetric with respect to electron exchange. [Atkins and Friedman, 1997] This was addressed by J. C. Slater and V. Fock by including spin orbitals and the effect of electron exchange. They stated that the product of the molecular orbital and spin function must not be neglected and that it is essential to take linear combinations of anti-symmetric products of spin orbitals. This type of self-consistent field calculations 
received the name Hartee-Fock calculation. [Atkins and Friedman, 1997]

In this thesis, restricted calculations were performed, that is, calculations of a closed shell nature to analyze the excited states of glyoxal. Two different types of basis sets were used in these calculations. Below is a discussion, which describes basis sets used for the calculations.

Electron spin

All electrons have either of two types of spin, up $(+1 / 2)$ or down $(-1 / 2)$. Molecular calculations can either be closed shell or open shell. Closed shell calculations are referred to as restricted calculations and used doubly occupied orbitals that hold two electrons having opposite spins. Open shell calculations, better known as unrestricted calculations, use separate orbitals for the spin up and the spin down electrons. The two spin functions, $\alpha$ and $\beta$, are defined as follows:

$$
\begin{array}{ll}
\alpha(+1 / 2)=1 & \alpha(-1 / 2)=0 \\
\beta(+1 / 2)=0 & \beta(-1 / 2)=1
\end{array}
$$

The function is 1 when an electron is spin up and the $\beta$ function is 1 when the electron is spin down. The notation 
$\alpha(i)$ and $\beta(i)$ designate the values of $\alpha$ and $\beta$ for electron i. To include the electron spin in the electron wavefunction, the molecular orbital function is multiplied by either $\alpha$ or $\beta$. The resultant product of the molecular orbital in a spin orbital is defined as a spin orbital, a function of both the electron's location and it's spin.

\section{Basis sets}

A basis set is the mathematical description of the orbitals within a system (which in turn combine to approximate the total electronic wavefunction), used to perform the theoretical calculation. [Foresman and Frisch, 1996] In general, the larger the basis set the more accurate the approximation to the orbitals becomes.

Standard basis sets express the molecular orbitals as linear combinations of a predefined set of one-electron functions known as basis functions. These basis functions are usually centered on the atomic nuclei and so resemble atomic orbitals. Each individual molecular orbital is defined as:

$$
\phi_{i}=\sum c_{u i} \chi_{\mu}
$$

where the coefficients $c_{\mu i}$ are known as the molecular orbital expansion coefficients. The basis functions, $\chi_{1} \ldots \chi_{N}$, 
are chosen to be normalized. Moreover, $\chi_{\mu}$ refers to an arbitrary basis set in the same way, $\phi_{i}$, refers to an arbitrary molecular orbital. There are two types of commonly used basis functions: Slater-type orbitals (STOs) and Gaussian-type orbitals (GTOs).

Gaussian-type functions are used as basis functions in the Gaussian suite of programs and are defined as follows [Atkins and Freidman, 1997]:

$$
g(\alpha, r)=c x^{n} y^{m} z^{1} e^{-\alpha r^{2}}
$$

where $r$ is the position vector $(x, y, z)$ and $\alpha$ is a constant determining the size (radial extent) of the function. In a Gaussian function, $e^{-\alpha r^{2}}$ is multiplied by powers (possibly 0 ) of $x, y, z$ and a constant for normalization, so that, $\int g^{2}=1$. The three gaussian functions (s, py and dxy types, respectively) are:

$$
\begin{array}{ll}
g_{s}(\alpha, r)=(2 \alpha / \pi)^{3 / 4} e^{-\alpha r^{2}}, & (4.10 a) \\
g_{y}(\alpha, r)=\left(128 \alpha^{5} / \pi^{3}\right)^{1 / 4} y e^{-\alpha r^{2}}, & (4.10 \mathrm{~b}) \\
g_{x y}(\alpha, r)=\left(2048 \alpha^{7} / \pi^{3}\right)^{1 / 4} \mathrm{xye}^{-\alpha r^{2},} & (4.10 \mathrm{c})
\end{array}
$$


Linear combinations of primitive gaussian like these are used to form the actual basis function; the latter are called contracted Gaussians and have the form [Foresman and Frisch, 1996]:

$$
\chi_{\mu}=\sum \mathrm{d}_{\mu \mathrm{p}} \mathrm{g}_{\mathrm{p}}
$$

where the $d_{\mu p}$ 's are fixed constants within a given basis set. This results in the following expansion for molecular orbitals:

$$
\phi_{i}=\sum c_{\mu i} \chi_{\mu}=\sum c_{\mu i}\left(\sum d_{\mu p} g_{p}\right)
$$

\section{Basis set effects}

Minimal basis sets contain the minimum number of basis functions needed for each atom. A way to improve the minimal basis set is to increase the number of basis sets per atom. Split valence basis sets, such as 3-12G and 631G, have two (or more) sizes of basis function for each valence orbital. The adopted nomenclature is a guide to the contraction scheme. The first number indicates the number of primitives used in the contracted core function, while the numbers after the hyphen indicate the number of primitives used in the valence functions; e.g. if there are 
two such numbers, it is a valence-double-zeta basis, if there are three, valence-triple-zeta. [Cramer, 2002]

Atomic orbitals can change in size and shape depending on the neighboring atom when a bond is formed. In order to account for the distortions caused by bond formations, basis functions can be included to represent the orbitals of higher values of 1 . The distortion of a 1s-orbital can be described by the addition of a p-type basis function, and a d-type function can describe distortions of porbitals. Thus, 6-31G(d) indicates that it is the 6-31G basis set with d-type functions added to heavy atoms. Along the same lines, the polarized basis set $6-31 G(d, p)$ adds p-type functions to hydrogen atoms in addition to the d-type functions on heavy atoms.

\section{Diffuse functions}

Diffuse functions, also known as augmented functions (designated by + or ++ ), allow orbitals to occupy a larger region of space. The $6-31+G(d)$ basis set is the $6-31 G(d)$ basis set with diffuse functions added to heavy atoms while the double plus version, $6-31++G(d)$, adds diffuse functions to the hydrogen atoms as well.

Basis sets with diffuse functions are important for systems where electrons are relatively far from the nucleus, i.e. systems in their excited states. [Foresman and Frisch, 
1996] Since the goal of this thesis was to model the excited spectra of glyoxal, the $6-31+G(d)$ seemed the logical basis set of choice. For better accuracy, the triple-zeta basis set was used as well. This was done in the hopes of increasing the flexibility of the representative glyoxal molecule and to improve the outer valence region.

\section{Electron correlation}

It is common knowledge that the Hartree-Fock theory provides an inadequate treatment of the correlation between the motion of the electrons within a molecular system, especially those between electrons of opposite spin. When Hartree-Fock theory fulfills the requirement that $|\Psi|^{2}$ be invariant with respect to the exchange of any two electrons by anti-symmetrizing the wavefunction, it automatically includes the major correlation effects arising from pairs of electrons with the same spin. [Foresman and Frisch, 1996] This is better known as exchange correlation. Under Hartree-Fock theory, however, the motion of electrons of opposite spin remains uncorrelated. A method such as configuration interaction, which goes beyond SCF in an attempt to treat this observable fact properly, is known as an electron correlation method or a post-SCF method. 
Configuration interaction

Configuration interaction (CI) methods begin by noting that the exact wavefunction cannot be expressed as a single determinant, as Hartree-Fock theory assumes. [Foresman and Frisch, 1996] CI proceeds by constructing other determinants by replacing one or more occupied orbitals within the Hartree-Fock determinant with a virtual orbital. [Foresman and Frisch, 1996] In a single substitution, a virtual orbital replaces an occupied orbital within the determinant. This is equivalent to exciting an electron to a higher energy orbital. In the same manner, in a double substitution, $\phi_{a} \leftarrow \phi_{i}$ and $\phi_{b} \leftarrow \phi_{j}$, two occupied orbitals are replaced by virtual orbitals, for example:

$$
\Psi_{\text {ia }}=\left|\phi_{1}, \ldots \phi_{i}, \phi_{a+1}, \ldots \phi_{i-1}, \phi_{a}, \ldots \phi_{n}\right|
$$

Similarly, triple substitutions would exchange three orbitals, and so on. The full CI method forms the wavefunction $\psi$ as a linear combination of the Hartree-Fock determinant and all possible substituted determinants;

$$
\psi=\mathrm{c}_{\circ} \psi_{\mathrm{HF}}+\mathrm{c}_{1} \psi_{1}+\mathrm{c}_{2} \psi_{2}+\ldots
$$


where the coefficients c reflect the weight of each determinant in the expansion and also ensures normalization.

Although the full CI method is well defined, sizeconsistent, and variational it is also very expensive and impractical for all but very small systems. Practical CI methods augment the Hartree-Fock by adding only a limited set of substitutions. Such methods will truncate the CI expansion at some level of substitution, for example, the CIS method adds single excitations to the Hartree-Fock determinant, CID adds double excitations, CISD adds singles and doubles, CISDT adds singles, doubles, and triples, and so on. [Foresman and Frisch, 1996]

One way to think about the Hartree-Fock process is that it is an optimization of orbitals subject to the constraint that single excitations do not contribute to the wavefunction. [Cramer, 2002] The CI-singles (CIS) method, on the other hand, can be useful for excited states. Some other methods used in the analysis of excited states are the complete active space multiconfigurational selfconsistent field (CASSCF) method and the time-dependent Hartree-Fock or DFT method. Since only singlet excitations were of interest in the analysis of the near UV absorption spectrum of glyoxal, this was the method of choice. 
The tool used to perform the computational calculations for this thesis Gausian. Gausian is a system of programs for performing a variety of semiempirical and $a b$ initio molecular orbital (MO) calculations. [Friedman and Frisch, 1999] The version of Gaussian used is the Gaussian 98 software. Gaussian 98 is capable of predicting many properties of molecules and reactions, including: 1) molecular energies and structures, 2) energies and structures of transition states, 3) vibrational frequencies, 4) IR and Raman spectra, 5) thermochemical properties, 6) bond and reaction energies, 7) reaction pathways, 8) molecular orbitals, 9) atomic charges, 10) multiple moments, 11) NMR sheilding and magnetic susceptibilities, 12) vibrational circular dichronism intensities, 13) electron affinities and ionization potentials, 14) polarizabilities a n d hyperpolarizabilities, and 15) electrostatic potentials and electron densities. [Freidman and Frisch, 1999] Gaussian 98 was instrumental in the course of this research to help model a portion of the continuous spectrum of glyoxal in the near-UV region, that is, 200-350 nm.

\section{Gaussian input}

Gaussian is known as a user-friendly program. It is 
Outline of Gaussian Input

Input Section

Link 0 Commands

* Route Section

*blank line

*Title section

*blank line

*Molecule

Specification

*blank line

variables section

blank line
Contents

Defines the locations of scratch files and job resource limits

specifies the job type and model chemistry

Separates the route section from the title section

Describes the job for the output and archive entry

Gives the structure of the molecule to be studied

Specifies values for the variables used in the molecule specification

also flexible and allows the usage of a variety of different tasks to be performed without the necessity of making changes to the overall structure of the program.

Gaussian input has the basic structure outlined in Table 4.1. Note that the input sections marked with an asterisk are required in every input file. [Friedman and Frisch, 1996]

Gaussian accepts molecule specifications in several different formats, for example, Cartesian coordinates and z-matrix format (internal coordinates). Appendix B has a 
detailed explanation of the Gaussian input file sections including, as an example an interpretation of one of the jobs performed for the purposes of this research. 


\section{Chapter V}

Before reporting the results from the molecular calculations performed a brief discussion is dedicated to group theory. Also reported is the determination of transglyoxal's representations of its fundamental vibrational frequencies by applying group theory.

\section{Group Theory}

symmetry and group theory can be powerful tools for studying bonding and spectroscopy. In fact, properties such as measurement of crystal structures, infrared spectra, ultra-violet spectra, dipole moments, and optical activities depend on molecular symmetry.

The basis for our understanding of molecular structure (rather than simply its determination) lies in quantum mechanics and, therefore, any consideration of the role of symmetry in chemistry is basically a consideration of its role in quantum mechanics. [Bishop, 1973] The link between symmetry and quantum mechanics is provided by that part of mathematics known as group theory.

For the purposes of molecular spectroscopy, it is routinely assumed that the internal energy of a system can be expressed as the sum of rotational, vibrational, and electronic contributions [Carter, 1998]: 


$$
E_{\text {int }}=E_{x}+E_{v}+E_{e}
$$

This implies the overall wavefunction is:

$$
\Psi=\psi_{\mathrm{r}} \psi_{\mathrm{v}} \psi_{\mathrm{e}}
$$

for which the individual energies are given by separate Schrodinger equations of the form $\mathrm{H} \Psi=\mathrm{E} \Psi$. Solution to these equations is greatly simplified by changing the coordinates of the nuclei from cartesian coordinates to a new type, defined in a special way, called the normal coordinates.

In group theory, each normal coordinate belongs to one of the irreducible representations of the point group of the molecule concerned and it is a part of a basis, which can be used to produce that representation. The vibrational wavefunctions associated with the fundamental vibrational energy levels behave in the same way because of their relationship with the normal coordinates. [Bishop, 1973] It is, therefore, possible to classify both the normal coordinates and fundamental vibrational wavefunctions according to their symmetry species and to predict from the character tables the degeneracies and symmetry types which can, in principle, exist. [Bishop, 1973 ] Moreover, knowledge of the irreducible 
representations to predict from the vibrational wavefunctions reveals a good deal about the spectra of the molecule under consideration.

\section{Molecular vibrations}

The mass weighted displacements of the nuclei of a molecule from their equilibrium position, $\mathrm{q}_{\mathrm{I}}$, can be used to generate a representation of the point group to which the molecule belongs. [Bishop, 1973] Under the symmetry operation, $R$, the mass weighted nuclear displacements $q_{1}$, $q_{2}, \ldots q_{3 N}$ become $q_{1}^{\prime}, q_{2}^{\prime}, \ldots q_{3 N}^{\prime}$ then;

$$
\mathrm{q}_{i}^{\prime}=\sum \mathrm{D}_{i j}^{\circ}(R) \mathrm{q}_{j}
$$

where $i=1,2, \ldots 3 \mathrm{~N}$. The matrices $D^{\circ}(R)$ contain $3 \times 3$ block matrices, one for each atom. For any operation $R_{i}$, all the individual-atom operator matrices ( $3 \times 3$ matrices) have identical form and therefore, have the same character, $\chi_{i}$. [Carter, 1998] Only individual-atom block matrices that lie along the diagonal of the full operator matrix contribute to the overall operation, $\chi_{R}$. Furthermore, only those $3 \times 3$ block matrices for the atoms that are not shifted by the operation contribute to the character of the overall matrix. Thus, to find $\chi_{R}$, the number of atoms that remain 
non-shifted by the operation are counted, $\mathbf{N}_{i}$, and multiplied by the contribution per non-shifted atom, $\chi_{I}$, that is;

$$
\chi_{\mathrm{R}}=\mathrm{N}_{\mathrm{i}} \chi_{\mathrm{i}}
$$

where $\chi_{i}$ is equivalent to the character of the $3 \times 3 \mathrm{block}$ matrices of which the full matrix of the operation is composed. (Carter, 1998)

In an examination of the individual-atom transformation matrices comprising the full matrix for an operation, the individual elements $c_{11}, c_{22}, c_{33}$ (along the trace of the $3 \times 3$ matrix) indicate the effects of the operation on the coordinates $x, y, z$, respectively, on the atoms on which they operate. Therefore, the values for these elements will be identical to the characters for the operation in the irreducible representations by which the unit vectors $x, y, z$ transform. $\chi_{i}$, then, is simply attained by adding up the characters in the character table under the operation for the irreducible representations by which all the three unit vectors transform.

Fundamental vibrational frequencies for glyoxal

Table 5.1 incorporates this instruction needed to generate the reducible representation for the fundamental vibrational frequencies of glyoxal in the $\mathrm{C}_{2} \mathrm{~h}$ trans-glyoxal 
Table 5.1

Fundamental Frequencies for Trans-Glyoxal

\begin{tabular}{|c|c|c|c|c|c|}
\hline $\mathrm{C}_{2} \mathrm{~h}$ & $E$ & $\mathrm{C}_{2}$ & $i$ & $\sigma_{\mathrm{h}}$ & \\
\hline $\mathrm{N}_{\mathrm{i}}$ & 6 & 0 & 0 & 6 & \\
\hline$\chi_{i}$ & 3 & -1 & -3 & 1 & \\
\hline$\Gamma^{3 \mathrm{~N}}$ & 18 & 0 & 0 & 6 & $\Sigma$ \\
\hline$A_{g}$ & 18 & 0 & 0 & 6 & 24 \\
\hline $\mathbf{B}_{g}$ & 18 & 0 & 0 & -6 & 12 \\
\hline$A_{u}$ & 18 & 0 & 0 & -6 & 12 \\
\hline $\mathbf{B}_{u}$ & 18 & 0 & 0 & 6 & 24 \\
\hline
\end{tabular}

configuration. It also notes the transformation properties of the unit vectors and rotational vectors from the character table. It shows that:

$$
\Gamma_{3 \mathrm{~N}}=6 \mathrm{~A}_{\mathrm{g}}+3 \mathrm{~B}_{\mathrm{g}}+3 \mathrm{~A}_{\mathrm{u}}+6 \mathrm{~B}_{\mathrm{u}}
$$

There are, however, $3 N-6=12$ normal modes of vibration. The symmetry species of the genuine normal modes of vibration are identified by removing the species for the three translations and three rotations the molecule processes from $\Gamma_{3 N}$, that is, 


$$
\Gamma_{3 \mathrm{~N}-6}=\Gamma_{3 \mathrm{~N}}-\Gamma_{\text {trans }}-\Gamma_{\mathrm{rot}}
$$

For trans-glyoxal, $\Gamma_{\text {trans }}=A_{u}+2 B_{u}$ and $\Gamma_{\text {rot }}=A_{g}+2 B_{g}$. Applying Equation (5.6) gives;

$$
\Gamma_{3 \mathrm{~N}-6}=5 \mathrm{~A}_{\mathrm{g}}+\mathrm{B}_{\mathrm{g}}+2 \mathrm{~A}_{\mathrm{u}}+4 \mathrm{~B}_{\mathrm{u}}
$$

corresponding to the normal vibrational modes for transglyoxal.

\section{Allowed transitions}

As mentioned previously, it is usually assumed that the internal energy of a molecule can be decomposed into rotational, vibrational and electronic components. The transition between an electronic ground state $\psi_{e}$ and some excited state $\psi_{e}^{\prime}$ will be observable as a band in the absorption spectrum if there is a nonzero transition moment of the form:

$$
M_{e}=\int \psi_{e} \mu \psi_{e}^{\prime} d \tau
$$

in which $\mu$ is the electronic dipole moment operator whose components resolve as $\mu=\mu_{\mathrm{x}}+\mu_{\mathrm{y}}+\mu_{\mathrm{z}}$.

Transitions that occur because the electronic wave functions are not fully independent of the vibrational 
functions and the integral in Equation (5.9) can take on the form:

$$
\int\left(\psi_{\mathrm{e}} \psi_{\mathrm{v}}\right) \mu\left(\psi_{\mathrm{e}}^{\prime} \psi_{\mathrm{v}}^{\prime}\right) \mathrm{d} \tau
$$

Because the vibrational ground state is totally symmetric, however, $\psi_{\mathrm{e}} \psi_{\mathrm{v}}$ has the same symmetry as $\psi_{\mathrm{e}} \cdot \psi_{\mathrm{v}}{ }^{\prime}$, on the other hand, may have the symmetry of any of the normal modes.

If any transition has the appropriate symmetry so that the direct product, $\psi_{\mathrm{e}} \mu \psi_{\mathrm{e}}^{\prime} \psi_{\mathrm{v}}^{\prime}$, contains the totally symmetric representation then the integral will not vanish, that is it is allowed. [Carter, 1998] A similar analysis for trans-glyoxal, $C_{2} h$, illustrates that the transition $A_{u} \leftarrow A_{g}$ has the integral:

$$
\Gamma^{A g} \Gamma^{x y z} \Gamma^{A u}=A_{g}+2 B_{g}
$$

which contains the symmetric representation $A_{g}$. Thus, the transition $A_{u} \leftarrow A_{g}$ is allowed. 


\section{Chapter VI}

As mentioned in chapter I, the most characteristic electronic feature of the glyoxal molecule are the free oxygen non-bonding $(n)$ orbitals and the $\pi$-orbitals on the $C=0$ groups. Upon excitation of the glyoxal molecule, the $A_{u} \leftarrow A_{g}$ and $B_{g} \leftarrow B_{u}$ configurations are allowed whereas $B_{g} \leftarrow A_{g}$ and $A_{u} \leftarrow B_{u}$ are forbidden by symmetry.

$\mathrm{Ab}$ initio calculations can be useful for guiding the assignment of vibrational spectroscopy. Using the CIS method, the identification of the vibration observed in the continuous area of the glyoxal spectrum at the $280 \mathrm{~nm}$ wavelength is described below.

\section{Analysis of Gaussian calculations}

Molecular systems have stable, higher energy electronic configurations known as excited states. Such states are produced, for example, when a sample is exposed to the light source of a UV/Visible spectrophotometer. Modeling excited states and predicting their properties is a difficult problem; a theoretical method must avoid always ending up at the ground state. [Foresman and Frisch, 1996] The CI approach, named CI-Singles, models excited states as combinations of single substitutions out of the HartreeFock (HF) ground state. CI-Singles is described by its 
developers as "an adequate zeroth-order treatment for many of the excited states of molecules," and in this sense is comparable to $\mathrm{HF}$ theory for ground state systems in that it is qualitatively accurate if not always highly quantitatively predictive. [Foresman and Frisch, 1996]

A more accurate method to study excited state system is the complete active space multiconfigurational selfconsistent field (CASSCF). This method uses a combination of an SCF computation with a full configuration interaction calculation involving a subset of the orbitals.

The time-dependent (TD) method keyword in Gaussian can also be useful in excited state energy calculations using the Hartree-Fock or DFT method. In a TDDFT method, KohnSham orbital energies and various exchange integrals are used in place of matrix elements of the Hamiltonian. [Cramer,2001] TDDFT results are most reliable when, i) the excitation energy is significantly smaller than the molecular ionization potential and ii) promotion(s) take place into orbitals having positive Kohn-Sham eigenvalues. [Cramer, 2001]

The Gaussian 98 cIS feature affords the ability to optimize a geometry and perform frequency calculations for systems in an excited state. Using Gaussian 98, CIS, the first, second and third excited states of glyoxal were 
located via an energy calculation. An optimization was then performed, starting from that point. Finally, frequencies were calculated at the optimized geometry. See Appendix B for a detailed explanation of the step job route section.

\section{Job analysis}

When requesting for the optimization of an excited state for glyoxal using the CIS method, the Gaussian output gives the energies, excitation and oscillator strengths of each computed excited state. For the second excited state analysis, the same input file as for the first excited state was used, only with the specified Root=2 in the route section. Root $=3$ was requested for the third excited state.

\section{First excited state}

The results for the frequency calculations of the DZP CIS level of theory for the first excited state of glyoxal are shown in Table (6.1). Listed are the vibrational frequencies and their corresponding motion. These motions were verified through a software package called Molden.

\section{Molden}

Molden can animate reaction paths as well as molecular vibrations from Gaussian run output files. It is a package for displaying molecular density from $a b$ initio packages which includes the Gaussian 98 software. Molden reads all 


\title{
Table 6.1
}

\begin{abstract}
Results of the Frequency $\left(\mathrm{cm}^{-1}\right)$ Calculations for the First Excited State of Trans-Glyoxal Using the CIS Method
\end{abstract}

\section{Basis set}

Trans isomer

$v_{1} \quad \mathrm{a}_{\mathrm{g}} \quad \mathrm{CH}$ stretch

$v_{2} \quad a_{\mathrm{g}} \quad$ CO stretch

$\begin{array}{lll}v_{3} & \mathrm{a}_{\mathrm{g}} & \mathrm{CH} \text { rock }\end{array}$

$v_{4} \quad a_{g} \quad$ CC stretch

$v_{5} \quad a_{g} \quad$ CCO bend

$\begin{array}{lll}v_{6} & \mathrm{a}_{\mathrm{u}} & \mathrm{CH} \text { wag }\end{array}$

$\begin{array}{lll}v_{7} & a_{u} \text { Torsion }\end{array}$

$v_{8} \quad b_{g} \quad \mathrm{CH}$ wag

$v_{9} \quad b_{u} \quad \mathrm{CH}$ stretch

$v_{10} \quad b_{u} \quad$ CO stretch

$v_{11} \quad b_{u} \quad \mathrm{CH}$ rock

$v_{12} \quad b_{u} \quad$ CCO bend
$6-31+G(D)$

${ }^{1} A_{u}$ state

3187.6

1876.5

1395.2

1238.3

626.4

805.4

263.0

905.4

3179.8

1846.8

1352.8

259.1

the required information from the Gaussian output file. It can write a variety of graphics formats; (postscript, XWindows, VRML, povray, OpenGL, teckronics 4014, hpgl, hp 23929, and Figure).

The XWindows version of Molden is also capable of importing and displaying chemx, $\mathrm{PDB}$, and a variety of mopac/ampac files and lots of other formats. [http://www.cmbi.kun.nl/-schaft/molden/molden.html] The zmatrix format was the format used in the computational analysis of, this thesis. 
Evaluation of CIS performance

Table 6.2 presents the $6-311++G(D, P)$ CIS frequency for trans-glyoxal in the $A_{u}$ electronic state along with fundamental frequencies that have been observed directly in, or inferred from, experiments. The o error for each vibrational mode is also listed.

While it is certainly true that a comparison between calculated harmonic frequencies and measured fundamental vibrational transition energies is imperfect, harmonic correlations for excited electronic states should generally be similar to those in the ground states. That is, fundamental frequencies are less than $10 \%$ below the corresponding harmonic values for stretching modes and within a few percent (with uncertain sign) for bending and torsional vibrations. [Stanton and Gauss, 1997]

It can be seen from Table 6.2 that the agreement between the theoretical and experimental frequencies in both electronic states is acceptable. Table 6.3 reports the results obtain by Stanton and Gauss for the theoretical harmonic frequencies for the $A_{g}$ and $A_{u}$ electronic states for trans glyoxal using the EOMEE-CCSD level of theory.

The EOMEE-CCSD method is similar in spirit to single reference configuration using the same determinantal basis, but with a straightforward application of perturbation 


\section{Table 6.2}

Results of the Frequency $\left(\mathrm{cm}^{-1}\right)$ Calculations for First Excited State of Trans-Glyoxal Using the 6-311++G(D,P)

CIS Method

Basis Set

Trans isomer

$v_{1} \quad a_{g} \quad \mathrm{CH}$ stretch

$v_{2} \quad a_{g} \quad$ CO stretch

$\begin{array}{lll}v_{3} & a_{9} & C H \\ \text { rock }\end{array}$

$v_{4} \quad a_{g} \quad$ CC stretch

$v_{5} \quad a_{g} \quad C C O$ bend

$\begin{array}{lll}v_{6} & a_{4} & C H \\ \text { wag }\end{array}$

$\begin{array}{lll}v_{7} & a_{u} & \text { Torsion }\end{array}$

$v_{8} \quad b_{g} \quad C H$ wag

$v_{9} \quad b_{u} \quad C H$ stretch

$v_{10} \quad b_{u} \quad$ CO stretch

$\begin{array}{lll}v_{11} & b_{u} & \mathrm{CH} \text { rock }\end{array}$

$v_{12} \quad b_{u} \quad$ CCO bend
6-311++G(D,P) Experimental \%error

' $A_{u}$ state

$3126.6 \quad 2809$

$1859.3 \quad 1391$

$1384.8 \quad 1195$

$1221.0 \quad 952$

$624.9 \quad 509$

$797.7 \quad 720$

$261.7 \quad 233$

$899.6 \quad 735$

3118.8

$1824.9 \quad 1281$

$1338.8 \quad 1172$

$251.8 \quad 379$
11.3

33.7

15.9

28.3

22.8

10.8

12.3

18.5

42.5

14.2

33.6

theory. [Stanton and Gauss, 1997] As noted in Table 6.3 EOMEE-CCSD is excellent for excited state descriptions, particular for those states that can be categorize as 'singly' excited states. The accuracy in performance can be attributed to its treatment of excited state electron correlation roughly comparable in quality to the corresponding ground state couple cluster method (CCSD) combined with computational scaling properties that allow its application to medium-sized molecules. [Stanton and Gauss, 1997] In comparison of Tables 6.2 and 6.3 , it should be noted that a better agreement is attained using 
Results by Stanton and Gauss [1997] for the Frequency Calculations for the Ground and First Excited states of

Trans-Glyoxal Using the EOMEE-CCSD Level of Theory

Basis Sets

$$
\text { 6-31+G(D) 6-311++G(D,P) Exp. }
$$

Trans

$\begin{array}{llll}v_{1} & a_{a} & C H & \text { stretch }\end{array}$

$\begin{array}{llll}v_{2} & a_{a} & \text { CO stretch }\end{array}$

$\begin{array}{llll}v_{3} & a_{a} & C H \\ \text { rock }\end{array}$

$\begin{array}{llll}v_{4} & a_{a} & C C & \text { stretch }\end{array}$

$\begin{array}{lll}v_{5} & a_{a} & \text { CCO bend }\end{array}$

$\begin{array}{llll}v_{6} & a_{u} & C H & \text { wag }\end{array}$

$v_{7} a_{u}$ Torsion

$\begin{array}{llll}v_{8} & b_{a} & C H & \text { wag }\end{array}$

$v_{9} \mathrm{~b}_{u} \quad \mathrm{CH}$ stretch

$\begin{array}{llll}v_{10} & b_{u} \quad \text { CO stretch }\end{array}$

$v_{11} b_{u} \quad \mathrm{CH}$ rock

$\begin{array}{llll}v_{12} & b_{u} & \text { CCO bend }\end{array}$

$$
{ }^{1} A_{g} \text { state }
$$

3057.2

1851.5

1418.1

1117

567.3

837.3

145.7

1072.7

3052.2

1809.7

1372.5

338.1

$\begin{array}{rc}3042.3 & 2843 \\ 1821.6 & 1745 \\ 1413.2 & 1338 \\ 1097.5 & 1065 \\ 562.8 & 551 \\ 821.8 & 801 \\ 126 & 127 \\ 1081.3 & 1048 \\ 3035.9 & 2835 \\ 1793.4 & 1732 \\ 1373 & 1312 \\ 340.8 & 339\end{array}$

Basis Sets 
inferred internuclear separation is shorter than that predicted by calculation and thus, it appears that mode is relatively difficult to describe theoretically. [Stanton and Gauss, 1997]

Second excited state

For the second excited state, as stated earlier, he input file was the same as for the first excited state, except for the specified $R O O T=2$ in the route section. The results are given in Table 6.4 .

In the output for the second excited state of transglyoxal, the transition $B_{g} \leftarrow A_{g}$ is observed. Inspection of the output reveals that the singlet $B_{q}$ state has an oscillator strength of 0.0000 , which means that it is a forbidden state and cannot be observed by single photon experiments, such as UV spectroscopy. [Foresman and Frisch, 1996] Any vibration, however, that distorts the molecule out of the $\mathrm{C}_{2} \mathrm{~h}$ symmetry will allow the molecule an excited state character.

It is known that geometry optimizations converge to a structure on the potential energy surface where the forces of the system reach equilibrium. Frequency calculations can be quite useful in disclosing the nature of a stationary point found by a geometry optimization. The final structure may correspond to a minimum on the 
Table 6.4

Results of the Frequency $\left(\mathrm{cm}^{-1}\right)$ Calculations for the
Second Excited State of Trans-Glyoxal Using the CIS
Method

Basis Sets

Trans isomer

$6-31+G(D) \quad 6-311++G(D, P)$

$\begin{array}{lllrr}v_{1} & a_{g} & \text { CH stretch } & 3214.2 & 3176.5 \\ v_{2} & a_{g} & \text { CO stretch } & 1977.7 & 1812.9 \\ v_{3} & a_{g} & \text { CH rock } & 1388.1 & 1325.6 \\ v_{4} & a_{g} & \text { CC stretch } & 994.9 & 1016.4 \\ v_{5} & a_{g} & \text { CCO bend } & 573.7 & 546.9 \\ v_{6} & a_{u} & \text { CH wag } & 653.6 & 832.9 \\ v_{7} & a_{u} & \text { Torsion } & -155.6 & 354.4 \\ v_{8} & b_{g} & \text { CH wag } & 812.3 & 850.4 \\ v_{9} & b_{u} & \text { CH stretch } & 3206.6 & 3173.9 \\ v_{10} & b_{u} & \text { CO stretch } & 1976.3 & 1702.3 \\ v_{11} & b_{u} & \text { CH rock } & 1322.2 & 1301.1 \\ v_{12} & b_{u} & \text { CCO bend } & -98.2 & 438.8\end{array}$

potential energy surface, or it may represent a saddle point, which is a minimum with respect to some directions on the surface and a maximum in one or more others. [Foresman and Frisch, 1996] First order saddle points, which are a maximum in exactly one direction and a minimum in all other orthogonal directions, correspond to transition sate structures linking two minima. [Foreman and Frisch, 1996] In the output of a frequency calculation imaginary frequencies are indicated as negative numbers. A 
structure which has $\mathrm{n}$ imaginary frequencies is an $\mathrm{nth}$ order saddle point. The frequency calculation of the optimized structure of the second excited state shows that the planar stationary point is not a minimum but has two imaginary vibrational frequencies. Whenever a structure yields an imaginary frequency, it means that there is some geometric distortion for which the energy of the system is lower than it is at the current structure (indicating a more stable structure).

First, for glyoxal the following processes are energetically possible at 308nm [Langford and Moore, 1984]:

$$
\begin{aligned}
(\mathrm{HCO})_{2}+\mathrm{hv} & \rightarrow \mathrm{CO}+\mathrm{CO}+\mathrm{H}_{2} \\
& \rightarrow \mathrm{H}_{2} \mathrm{CO}+\mathrm{CO} \\
& \rightarrow \mathrm{HCO}+\mathrm{HCO}
\end{aligned}
$$

indicating that any of these dissociations could possibly be taking place.

Secondly, the transition $B_{g} \rightarrow A_{g}$, although forbidden, indicates that the transformation to the molecular orbital representation $B_{g}$ facilitates any of the above dissociations. It should also be noted that the geometry optimizations of both the first and second excited states of trans-glyoxal show that the $\mathrm{CC}$ bonds are longer than in 
the ground state. (The second excited state optimized CC bond is longer than the first excited state by approximately 0.1 angstrom).

\section{Third excited state}

The results of the third excited state can be seen in Table 6.5. It reveals that there is one imaginary vibrational frequency.

Table 6.5

Results of the Frequency $\left(\mathrm{cm}^{-1}\right)$ Calculations for the Third Excited State of Trans-Glyoxal Using the CIS Method

Basis Sets

Trans isomer

$$
6-31+G(D) \quad 6-311++G(D, P)
$$

$\mathrm{CH}$ stretch

3218.8

3283.4

CO stretch

1759.1

1968.5

$\mathrm{CH}$ rock

1346.4

1356.9

1211.1

1230.4

CC stretch

392.8

394.2

618.6

$-1746.7$

695.4

620.0

$a_{u} \quad \mathrm{CH}$ wag

Torsion

$b_{g} \quad \mathrm{CH}$ wag

3189.4

$-1713.2$

704.0

$b_{u} \quad \mathrm{CH}$ stretch

1565.1

3256.4

$b_{u} \quad$ CO stretch

1331.5

1583.0

209.8

1347.7

215.8 
In 1981 , Osamura et al., predicted constrained $\mathrm{C}_{\mathrm{s}}$ stationary point geometries, possible transition states for the reaction:

$$
(\mathrm{CHO})_{2} \rightarrow \mathrm{H}_{2} \mathrm{CO}+\mathrm{CO}
$$

Their results are given in Table 6.6. They performed the calculations with the split valence $3-21 \mathrm{G}$ basis set, configuration interaction was carried out including all configurations differing by one or two orbitals from the SCF reference configuration. [Osamura et al., 1981] Examining Table 6.6, it appears that for the trans-glyoxal, the $3-21 \mathrm{G}$ basis contains a single imaginary vibrational frequency which is sizable, i.e. $1739 \mathrm{~cm}^{-1}$ and does, indeed, connect glyoxal with formaldehyde plus carbon monoxide. The similar imaginary frequency of this study and that obtained in Table 6.5 suggests that the predicted third excited state for the $6-311++G(D, P)$ CIS level of theory leads to the dissociation in Equation 6.2 .

\section{Spectral Analysis}

This section of the chapter incorporates the molecular calculations performed to the modeling of the glyoxal spectra experimentally obtained. The electronic absorption spectrum of a gas-phase molecule reveals electronic 
Results by Osamura et al. [1981] of the Vibrational Frequencies for the $C_{s}$ stationary Points Connecting Glyoxal with $\mathrm{H}_{2} \mathrm{CO}+\mathrm{CO}$

\section{Basis Set}

\section{$3-21 G$}

$\begin{array}{ll}v_{1} & a^{\prime} \\ v_{2} & a^{\prime} \\ v_{3} & a^{\prime} \\ v_{4} & a^{\prime} \\ v_{5} & a^{\prime} \\ v_{6} & a^{\prime \prime} \\ v_{7} & a^{\prime \prime} \\ v_{8} & a^{\prime} \\ v_{9} & a^{\prime} \\ v_{10} & a^{\prime} \\ v_{11} & a^{\prime \prime} \\ v_{12} & a^{\prime}\end{array}$

$3200 \mathrm{~cm}^{-1}$

2682

1984

1894

1308

1051

772

755

358

220

162

$-1739$

transitions consisting of a series of bands. Each band consists of a number of peaks. In the presence of a single progression, those peaks should have an approximately constant spacing in energy corresponding to a fundamental mode in the excited state. The range of the experimentally obtain spectra of glyoxal is from 200 to $350 \mathrm{~nm}$. This near-UV region characterizes a continuous portion of the absorption spectrum. Table 6.7 reports each peak's the spacings are an average of $1470 \mathrm{~cm}^{-1}$. 
Spacings for the Continuous Region of the Glyoxal spectra at the $280 \mathrm{~nm}$ Wavelength

$\begin{array}{crc}\text { Wavelength }(\mathrm{nm}) & \text { Wavenumbers }\left(\mathrm{cm}^{-1}\right) & \text { Spacing }\left(\mathrm{cm}^{-1}\right) \\ 255 & 39220 & \\ 264 & 37880 & 1340 \\ 275 & 36360 & 1520 \\ 287 & 34840 & 1520 \\ 298 & 33560 & 1280 \\ 311 & 32150 & 1410 \\ 328 & 30490 & 1660 \\ 346 & 28900 & 1590\end{array}$

Average

1470

Based on the energies obtained from the computational results, the second excited state transition is in progress in the region of study. Examination of Table 6.3 indicates that the only fundamental vibrational frequency that corresponds to the spacing value of $1470 \mathrm{~cm}^{-1}$ is the $A_{g} C O$ stretch. This, of course, is taking into account the overestimation characteristic of the CIS level of theory seen in the analysis of the first excited state.

The molecular orbital diagrams shown in Figure 1.4 (page 12) depict the fact that the transition to the excited electronic state molecular orbital representation 
introduces a node between the carbon and oxygen bond for the $A_{u} \leftarrow A_{g}$ transition. This configuration, in essence, facilitates a co stretch. In fact, the optimized structure of the excited state displays, as expected of the $\pi^{*} \leftarrow n$ type excitation process, the same effect; specifically, the co bond length is increased due to a reduction in bond order while the CC bond distance contracts and the CCO angle opens slightly. (Stanton and Gauss, 1997) Thus, given the frequency calculations obtained computationally, using the CIS method, and the above factors, the assignment of the $A_{g}$ Co stretch vibrational mode to the absorption peaks under study is evidently reasonable. 


\section{Chapter VII}

\section{Conclusion}

In this thesis, comprehensive, medium resolution absorption cross-sections have been reported for the $280 \mathrm{~nm}$ gas-phase absorption spectra of glyoxal at room temperature. The results are in good agreement with at least one set of previously reported values.

The results of this study will be of use in the determination of glyoxal quantum yields and ultimately the rates and products of photolysis, as well as serve as input for laboratory studies of glyoxal chemistry where glyoxal concentration is determined by absorption spectroscopy. In addition, this cross-section data can be used to estimate the lifetime of glyoxal with respect to photodissociation for a representative set of tropospheric conditions.

Using the CIS method, molecular orbital calculations were also performed, as a guide to identify the vibration responsible for the weak structure features of the continuous region of the glyoxal spectrum centered at the $280 \mathrm{~nm}$ wavelength. The vibration accountable for the progression of peaks was found to be the $A_{g}$ Co stretch vibration mode of the glyoxal molecule. 


\section{REFERENCES}

Atkins, P.W. and R.S. Freidman, Molecular Quantum Mechanics, Oxford University Press, New York, 1997.

Atkinson, G.H. and C.S. Parmenter, The $260 \mathrm{~nm}$ Absorption Spectrum of Benzene: Remeasured Band Positions and Redefined Assignments, J. Mol. Spec., 73, 20-30, 1978 .

Bishop David M., Group Theory and Chemistry, Dover Publications Inc., New York, 1973.

Bretherick, L., Handbook of Reactive Chemical Hazards, Butterworths, London, 1985. 1953.

Calvert, J.C. and G. Lane, J. Am. Chem. Soc., 75, 856,

Carpenter, C.I. and L.S. Forester, The spectra of Glyoxal Solutions, J. Phys. Chem., 62, 874-875, 1958 .

Carter, R.L., Molecular symmetry and Group Theory, John Wiley and Sons, Inc., New York, 1998.

Cramer, C.J., Essentials of Computatiuonal Chemistry: Theories and Models, Wiley, New York, 2002.

Drent, E. and J. Kommandeur, The Electronic States of Biacetyl, Chem. Phys. Letters, 14(3), 321-325, 1972.

Foresman, J.B. and A. Frisch, Exploring Chemistry with Electronic Structure Methods, Gaussian, Inc., Pittsburgh, PA, 1996.

Finlayson-Pitts, B.J. and J.N. Pitts, Jr., Chemistry of the Upper and Lower Atmosphere: Theory, Experiments and Applications, Academic Press, San Diego, CA, 2000.

Langford, A.O. and C.B. Moore, Collision complex formation in the reactions of formyl radicals with nitric oxide and oxygen, J. Chem. Phys., 80(9), 4211-4220, 1984 . 
Lewis, R.J., Hazardous Chemicals Desk Reference, John Wiley and Sons, Inc., New York, 1997.

Lui, Xiaoyu, H.E. Jeffries and K.G. Sexton, Atmospheric Photochemical Degradation of 1,4-Unsaturated Dicarbonyls, Environ. Sci. Technol., 33, 4212-4220, 1999.

Osamura, Yoshihiro and H.F. Schafer III, A unimolecular reaction $A B C \rightarrow A+B+C$ involving three product molecules and a single transition state. Photodissociation of glyoxal: $\mathrm{HCOHCO} \rightarrow \mathrm{H}_{2}+\mathrm{CO}+\mathrm{CO}$, J. Chem. Phys., 75(12), 5828-5836, 1981 .

Plum, C.N., E. Sanhueza, R. Atkinson, W.P.L. Carter and J.N. Pitts, Jr., OH Radical Rate Constants and Photolysis Rates of $\alpha$-Dicarbonyls, Environ. Sci. Technol., $17(8), 479-483,1983$.

Smith, D.F., T.E. Kleindienst and C.D. MCIver, Primary Product Distributions from the Reaction of OH with $m-, p-$ Xylene, 1,2,4- and 1,3,5-Trimethylbenzene, J. of Atmos. Chem., 34, 339-364, 1999.

Stanton, J.F and J Gauss, Theoretical study of electronically excited c is - and trans-glyoxal, Spectrochimica Acta Part A, 53, 1153-1162, 1997.

Volkamer, R., U. Platt and K. Wirtz, Primary and Secondary Glyoxal Formation from Aromatics: Experimental Evidence for the Bicycloalkyl-Radical Pathway from Benzene, Toluene and p-Xylene, J. Phys. Chem., 105, 7865-7874, 2001.

Zhu, Lei, D. Kellis and Chuan-Fan Ding, Photolysis of glyoxal at 193, 248, 308 and 351 nm, Chem. Physics Letters, $257,487-491,1996$. 


\section{APPENDIX A}

Summary of Data

The experimental absorption cross-section data for the near UV absorption spectra of glyoxal is summarized below. The data is presented in tabular form, listing the wavelength (in $\mathrm{nm}$ ) and the absorption cross-section (in $\mathrm{pm}^{2}$, where $1 \mathrm{pm}^{2}=10^{-20} \mathrm{~cm}^{2}$ ).

$\begin{array}{rr}350 & 0.01564 \\ 349.5 & 0.02538 \\ 349 & 0.05358 \\ 348.5 & 0.13615 \\ 348 & 0.24919 \\ 347.5 & 0.30048 \\ 347 & 0.28811 \\ 346.5 & 0.31363 \\ 346 & 0.3535 \\ 345.5 & 0.32572 \\ 345 & 0.28266 \\ 344.5 & 0.26619 \\ 344 & 0.23847 \\ 343.5 & 0.17926 \\ 343 & 0.14051 \\ 342.5 & 0.13472 \\ 342 & 0.14847 \\ 341.5 & 0.16896 \\ 341 & 0.17032 \\ 340.5 & 0.17725 \\ 340 & 0.21211 \\ 339.5 & 0.23288 \\ 339 & 0.23907 \\ 338.5 & 0.25427 \\ 338 & 0.2448 \\ 337.5 & 0.23178 \\ 337 & 0.22568 \\ 336.5 & 0.23339 \\ 336 & 0.26611 \\ 335.5 & 0.31397 \\ 335 & 0.39632 \\ 334.5 & 0.4873\end{array}$




$\begin{array}{rr}334 & 0.56436 \\ 333.5 & 0.60405 \\ 333 & 0.60472 \\ 332.5 & 0.62374 \\ 332 & 0.68699 \\ 331.5 & 0.77423 \\ 331 & 0.93282 \\ 330.5 & 1.15408 \\ 330 & 1.32369 \\ 329.5 & 1.42842 \\ 329 & 1.49908 \\ 328.5 & 1.53919 \\ 328 & 1.52476 \\ 327.5 & 1.45158 \\ 327 & 1.37655 \\ 326.5 & 1.31513 \\ 326 & 1.25559 \\ 325.5 & 1.22333 \\ 325 & 1.24635 \\ 324.5 & 1.29301 \\ 324 & 1.32324 \\ 323.5 & 1.3428 \\ 323 & 1.37977 \\ 322.5 & 1.4146 \\ 322 & 1.4409 \\ 321.5 & 1.48229 \\ 321 & 1.49616 \\ 320.5 & 1.48473 \\ 320 & 1.48237 \\ 319.5 & 1.51076 \\ 319 & 1.5876 \\ 318.5 & 1.72888 \\ 318 & 1.91212 \\ 317.5 & 2.04319 \\ 317 & 2.11299 \\ 316.5 & 2.19335 \\ 316 & 2.2792 \\ 315.5 & 2.3847 \\ 315 & 2.56855 \\ 314.5 & 2.7446 \\ 314 & 2.8549 \\ 313.5 & 2.94952 \\ 313 & 3.01892 \\ 312.5 & 3.01534 \\ 312 & 2.96134 \\ 311.5 & 2.89435 \\ 311 & 2.81531 \\ 310.5 & 2.75617\end{array}$




$\begin{array}{rr}310 & 2.74471 \\ 309.5 & 2.7495 \\ 309 & 2.75593 \\ 308.5 & 2.77542 \\ 308 & 2.80049 \\ 307.5 & 2.81729 \\ 307 & 2.83684 \\ 306.5 & 2.85835 \\ 306 & 2.85575 \\ 305.5 & 2.85689 \\ 305 & 2.88704 \\ 304.5 & 2.94105 \\ 304 & 3.01694 \\ 303.5 & 3.11971 \\ 303 & 3.23094 \\ 302.5 & 3.32468 \\ 302 & 3.41257 \\ 301.5 & 3.4954 \\ 301 & 3.54625 \\ 300.5 & 3.60104 \\ 300 & 3.67221 \\ 299.5 & 3.70997 \\ 299 & 3.72545 \\ 298.5 & 3.72408 \\ 298 & 3.68107 \\ 297.5 & 3.59999 \\ 297 & 3.51686 \\ 296.5 & 3.47282 \\ 296 & 3.45439 \\ 295.5 & 3.42996 \\ 295 & 3.39895 \\ 294.5 & 3.36069 \\ 294 & 3.31263 \\ 293.5 & 3.28709 \\ 293 & 3.2783 \\ 292.5 & 3.26437 \\ 292 & 3.2633 \\ 291.5 & 3.25929 \\ 291 & 3.26482 \\ 290.5 & 3.29443 \\ 290 & 3.34162 \\ 289.5 & 3.38333 \\ 289 & 3.40547 \\ 288.5 & 3.41798 \\ 288 & 3.41674 \\ 287.5 & 3.40681 \\ 287 & 3.39913 \\ 286.5 & 3.3797\end{array}$


$286 \quad 3.35101$

$285.5 \quad 3.29436$

$285 \quad 3.23676$

$284.5 \quad 3.18922$

$284 \quad 3.13912$

$283.5 \quad 3.07497$

$283 \quad 2.9985$

$282.5 \quad 2.94629$

$\begin{array}{ll}282 & 2.89384\end{array}$

$281.5 \quad 2.83296$

2812.78805

$280.5 \quad 2.75158$

$\begin{array}{ll}280 & 2.70438\end{array}$

$279.5 \quad 2.68132$

$279 \quad 2.66519$

$278.5 \quad 2.64876$

$\begin{array}{ll}278 & 2.64347\end{array}$

$277.5 \quad 2.64156$

$277 \quad 2.63833$

$276.5 \quad 2.61144$

$\begin{array}{ll}276 & 2.58096\end{array}$

$275.5 \quad 2.56133$

$275 \quad 2.53793$

$\begin{array}{ll}274.5 & 2.48997\end{array}$

$274 \quad 2.44821$

$273.5 \quad 2.40901$

$273 \quad 2.34464$

$\begin{array}{ll}272.5 & 2.28025\end{array}$

$\begin{array}{ll}272 & 2.22802\end{array}$

$\begin{array}{ll}271.5 & 2.17487\end{array}$

$\begin{array}{ll}271 & 2.11577\end{array}$

$270.5 \quad 2.06307$

$270 \quad 2.00456$

$269.5 \quad 1.95963$

$269 \quad 1.93067$

$268.5 \quad 1.90115$

$268 \quad 1.88113$

$267.5 \quad 1.86943$

$267 \quad 1.85995$

$266.5 \quad 1.84463$

$266 \quad 1.81855$

$265.5 \quad 1.79842$

$\begin{array}{ll}265 & 1.78592\end{array}$

$264.5 \quad 1.75947$

$264 \quad 1.73$

$263.5 \quad 1.69573$

$263 \quad 1.65402$

$262.5 \quad 1.59957$ 


$\begin{array}{rr}262 & 1.54213 \\ 261.5 & 1.49279 \\ 261 & 1.44362 \\ 260.5 & 1.40386 \\ 260 & 1.37352 \\ 259.5 & 1.34606 \\ 259 & 1.31386 \\ 258.5 & 1.28633 \\ 258 & 1.27658 \\ 257.5 & 1.26723 \\ 257 & 1.25248 \\ 256.5 & 1.23726 \\ 256 & 1.21107 \\ 255.5 & 1.18538 \\ 255 & 1.16854 \\ 254.5 & 1.14516 \\ 254 & 1.10151 \\ 253.5 & 1.05602 \\ 253 & 1.01873 \\ 252.5 & 0.98378 \\ 252 & 0.94512 \\ 251.5 & 0.89631 \\ 251 & 0.86514 \\ 250.5 & 0.84465 \\ 250 & 0.8201 \\ 249.5 & 0.80348 \\ 249 & 0.79451 \\ 248.5 & 0.78128 \\ 248 & 0.76942 \\ 247.5 & 0.75185 \\ 247 & 0.7276 \\ 246.5 & 0.70249 \\ 246 & 0.67948 \\ 245.5 & 0.65572 \\ 245 & 0.62078 \\ 244.5 & 0.59214 \\ 244 & 0.55583 \\ 243.5 & 0.53424 \\ 243 & 0.51691 \\ 242.5 & 0.49446 \\ 242 & 0.47621 \\ 241.5 & 0.45457 \\ 240.5 & 0.44916 \\ 239.5 & 0.414385 \\ & 0.37234\end{array}$




$\begin{array}{rr}238 & 0.36445 \\ 237.5 & 0.34772 \\ 237 & 0.31477 \\ 236.5 & 0.29247 \\ 236 & 0.28407 \\ 235.5 & 0.27268 \\ 235 & 0.26506 \\ 234.5 & 0.25609 \\ 234 & 0.24458 \\ 233.5 & 0.24723 \\ 233 & 0.24774 \\ 232.5 & 0.23511 \\ 232 & 0.2254 \\ 231.5 & 0.21625 \\ 231 & 0.21167 \\ 230.5 & 0.21 \\ 230 & 0.20344 \\ 229.5 & 0.19975 \\ 229 & 0.20141 \\ 228.5 & 0.20371 \\ 228 & 0.19764 \\ 227.5 & 0.20029 \\ 227 & 0.21675 \\ 226.5 & 0.22675 \\ 226 & 0.23043 \\ 225.5 & 0.2329 \\ 225 & 0.24098 \\ 224.5 & 0.25236 \\ 224 & 0.25592 \\ 223.5 & 0.26039 \\ 223 & 0.27516 \\ 222.5 & 0.29298 \\ 222 & 0.32098 \\ 221.5 & 0.35228 \\ 221 & 0.36482 \\ 220.5 & 0.38255 \\ 220 & 0.42322 \\ 219.5 & 0.45755 \\ 219 & 0.48891 \\ 218.5 & 0.53558 \\ 218 & 0.58146 \\ 217.5 & 0.63537 \\ 217 & 0.68982 \\ 216.5 & 0.74847 \\ 216 & 0.83482 \\ 215.5 & 0.93021 \\ 215 & 1.02616 \\ 214.5 & 1.13169\end{array}$


$\begin{array}{ll}214 & 1.24571\end{array}$

$213.5 \quad 1.36316$

$213 \quad 1.5051$

$212.5 \quad 1.67057$

$212 \quad 1.87116$

$211.5 \quad 2.14087$

$211 \quad 2.46174$

$210.5 \quad 2.8669$

$210 \quad 3.35795$

209.54 .01682

$209 \quad 4.57524$

$208.5 \quad 5.14656$

$208 \quad 5.5942$

$207.5 \quad 7.52821$

$207 \quad 8.00871$

$206.5 \quad 8.88536$ 


\section{APPENDIX B}

Gaussian Input File Format

Using as an example of the computational jobs performed to illustrate a Gaussian 98, a copy of an input file is given and its basic structure is described below.

Job File Input

$\% \mathrm{chk}=\mathrm{tst}$

\# RCIS/6-31+G(D) Test

glyoxal excited states

01

c

c 11.0

- 11.222120 .0

h 21.011120 .030 .0

h 111.022120 .044180 .0

o 21.221120 .03180 .0

--Link1--

schk=tst

$\because$ NoSave

\# RCIS(Root=1, Read) $/ 6-31+G(D)$ Opt Freq Geom=Check Guess=Read Test

frequencies

01

Route Section

Keyword (Option) Description

schk $=$ tst

\#RCIS
A check point file created by the program. Requests a cI-singles excited state calculation, preceded by $\mathrm{R}$ for restricted calculations. 
$6-31+G(D), 6-311++G(D, P)$ Describes the type of basis set.

Test

Commands Gaussian not to enter the results of the current job in the archive.

--Link1-- Starts a new job.

\&NoSave

Tells the program to delete the checkpoint file after the step concludes eventhough \&chk has been included.

RCIS(Root=n) Specifies which excited state is to be studied.

RCIS(Read) Reads the initial guesses for the CI-Singles states from the checkpoint file. This option is used to perform an additional job step for an excited state computed during the previous job step. It is accompanied by Guess=Read and Geom=Check.

Opt Tells the program to optimize the molecule at the level of theory described.

Freq Requests a frequency calculation.

Title section

The title section consists of one or more lines of descriptive information about the job. This section is terminated by a blank line. 
Specifying Molecular Structure

This section specifies the molecular structure of the system. Gaussian 98 accepts molecule specifications in different formats: 1) Cartesian coordinates, 2) z-matrix format (internal coordinates) and 3) mixed internal and Cartesian coordinates.

All the aforementioned formats require that the charge and the spin multiplicity of the system be specified first. "0" was used for the charge to represent a neutral transglyoxal molecule. The spin multiplicity is given by the equation $2 S+1$, where $s$ represents the total spin for the system. Since the restricted CIS was used, all the electrons are paired and have no contribution for the $\mathrm{s}$ (the $+1 / 2$ and $-1 / 2$ cancel each other). Consequently, the system has a multiplicity of "1".

For the description of each atom in the molecule, the z-matrix syntax was followed as the format of choice. It specifies the locations of, and bonds between atoms using bond lengths, bond angles, and dihedral (torsion) angles.

First, carbon 1 was selected as the starting atom in the molecule, making it the origin of the molecule in space. Secondly, another carbon atom (carbon 2) was connected to the first carbon atom, placing it along the $z-$ axis and specifying the length of the bond. Third, oxygen 
was chosen as the third atom bonded to carbon 1 by a distance of 2.0 and specifying the angel formed by the two bonds, that is, $120^{\circ}$.

Finally, the positions of all the subsequent atoms were specified by: 1) its atom label; 2) an atom to which it is bonded to and the bond length; 3) a third atom to it (or to the second atom) and the value of the resulting bond angle; 4) a fourth atom bonded to either end of the previous chain and the value of the dihedral (torsion) angle formed by the four atoms. Dihedral angles describe the angle the fourth atom makes with respect to the plane defined by the first three atoms; their values range from 0 to 360 degrees, or from -180 to 180 degrees. 